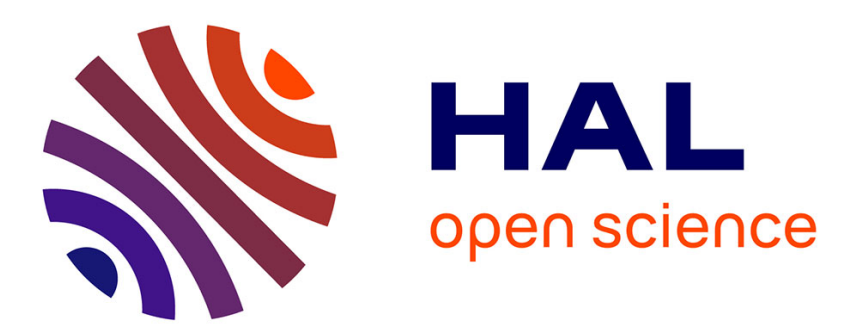

\title{
The Value of Network Information: Assortative Mixing Makes the Difference
}

Mohamed Belhaj, Frédéric Deroïan

\section{To cite this version:}

Mohamed Belhaj, Frédéric Deroïan. The Value of Network Information: Assortative Mixing Makes the Difference. 2016. halshs-01314954

\author{
HAL Id: halshs-01314954 \\ https://shs.hal.science/halshs-01314954 \\ Preprint submitted on 12 May 2016
}

HAL is a multi-disciplinary open access archive for the deposit and dissemination of scientific research documents, whether they are published or not. The documents may come from teaching and research institutions in France or abroad, or from public or private research centers.
L'archive ouverte pluridisciplinaire HAL, est destinée au dépôt et à la diffusion de documents scientifiques de niveau recherche, publiés ou non, émanant des établissements d'enseignement et de recherche français ou étrangers, des laboratoires publics ou privés. 


\section{amse}

école d'économie d'aix-marseille

aix-marseille school of economics

\section{Working Papers / Documents de travail}

\section{The Value of Network Information: Assortative Mixing Makes the Difference}

Mohamed Belhaj

Frédéric Deroïan 


\title{
The Value of Network Information: Assortative Mixing Makes the Difference*
}

\author{
Mohamed Belhaj ${ }^{\dagger}$ and Frédéric Deroïan ${ }^{\ddagger}$
}

May 11, 2016

$\dagger$ Centrale Marseille (Aix-Marseille School of Economics), CNRS and EHESS

$\ddagger$ Aix-Marseille University (Aix-Marseille School of Economics), CNRS and EHESS

\begin{abstract}
We study the value of network information in a context of monopoly pricing in the presence of local network externalities. We compare a setting in which all players, i.e. the monopoly and consumers, know the network structure and consumers' private preferences with a setting in which players only know the joint distribution of preferences, in-degrees and out-degrees. We give conditions under which network information increases profit or/and consumer surplus. The analysis reveals the crucial role played by four properties: degree assortativity, homophily (in preferences), preference-degree assortativity and preference-Bonacich centrality assortativity.
\end{abstract}

Keywords: Monopoly, Network Effects, Price Discrimination, Bonacich Centrality, Network Information, Degree Assortativity, Homophily, Preference-Degree Assortativity, Preference-Bonacich Centrality Assortativity.

JEL: C72, D85

*The authors thank the French National Research Agency (ANR) for their support through the program ANR 13 JSH1 0009 01. The authors are grateful for support from the A*Midex project (No. ANR-11-IDEX-0001-02) funded by the "Investissements d'Avenir" French Government program, managed by the French National Research Agency (ANR). E-mail addresses: mohamed.belhaj@centrale-marseille.fr, frederic.deroian@univ-amu.fr. 


\section{Introduction}

In many situations, consumption decisions are interdependent, shaped by network effects. To cite a few: online gaming, the membership to a social club, the access to video-conference technology, Dropbox use, any purchase based on recommendation by peers. Besides, numerical technologies today provide a huge amount of information about consumer characteristics, network relationships and consumer behaviors. This allows firms to exploit big data and use social network analysis softwares and services of Facebook, Twitter, Google, Klout, Toprankmarketing, etc, in order to target consumers. At the same time, consumers have better information about their relationships; for instance, Facebook or LinkedIn allow users to observe friends of friends, their purchase, etc. Incoming information can modify consumption decisions and firms' strategies and, therefore, profit and welfare. This raises the following question: is information about the network structure and consumer characteristics beneficial to firms and consumers?

In this paper we investigate the value of network information in the context of monopoly pricing under network externalities among consumers, in a model à la Candogan, Bimpikis and Ozdaglar (2012) (see also Bloch and Qérou [2013]). We compare the monopoly's profit and the consumer surplus under two settings. In the first, consumers and the monopolist are fully informed about the network structure and about consumer preferences for the good. In the second, consumers know their own preference for the good, their own in-degree and out-degree, as well as the joint distribution of these characteristics. The monopolist observes these characteristics for each consumer. ${ }^{1}$ From all the networks with the same joint degree distributions, we seek to identify those which generate a positive information value, i.e. an increase of profit and/or consumer surplus.

Under incomplete information, profit and consumer surplus can be expressed as functions of simple network statistics, whereas in the game of complete information, outcomes depend on Bonacich centralities. Because these centralities are complex objects, it is difficult to directly compare the outcomes of the two games. ${ }^{2}$ The first contribution of this paper is to establish an equivalence between the game of incomplete information and a modified game of complete information. In the modified game, each agent

\footnotetext{
${ }^{1}$ Hence, information rent is not an issue here. In contrast, Fainmesser and Galeotti (2015) study the value of information in a context of information asymmetry between a monopoly and consumers - in their settings, the consumers' information set is fixed while the monopoly's information set can vary.

${ }^{2}$ This difficulty has been pointed out by Fainmesser and Galeotti (2015): "There are no general results that map how adding or redistributing links in a network affects the Bonacich centralities of agents, and this is one of the reasons that the models of Bloch and Quérou (2013) and Candogan et al (2012) are less malleable in performing comparative statics."
} 
is influenced by all other agents, and the intensity of influence is equal to the ratio of the product of agents' in-degrees by neighbors' out-degrees to total number of links. Thus, rather than comparing the outcomes of the initial games directly, we compare the outcomes of two games of complete information, which are expressed as functions of Bonacich centralities.

Assortative mixing is a cornerstone of the analysis. A social network exhibits assortative mixing (Newman [2002]) if there is a positive correlation in the characteristics of people socially connected with each other. The characteristics can be a personal attribute (such as age, education, socio-economic status, physical appearance, and religion), or also a measure of centrality (e.g., degree, betweenness, Bonacich centrality, etc). Four types of assortativities will play a crucial role in the analysis. The first coefficient is the standard degree assortativity coefficient (Newman [2002]), which measures the likelihood of consumers with similar degrees to be linked with each other; we shall refer to degree assortativity when the coefficient is positive. The second one is the coefficient of assortative mixing by private preference, and measures the likelihood of consumers with similar preferences to be linked with each other (Newman [2003]); we will then speak about homophily when the likelihood is positive. ${ }^{3}$ The last two coefficients are novel and based on a generalization of assortative mixing to two characteristics. We call, by coefficient of preference-degree assortative mixing, the coefficient measuring the likelihood of consumers with high (resp. low) preferences to be linked with consumers of high (resp. low) degrees. We also call, by coefficient of preference-Bonacich centrality assortative mixing, the coefficient measuring the likelihood of consumers with high (resp. low) preferences to be linked with consumers of high (resp. low) Bonacich centrality. Our second contribution is to express those assortativity coefficients as functions of the difference between the interaction matrices of the game of complete information and the modified game of complete information.

Our third contribution is to link the value of information to assortative mixing. We start by analyzing the benchmark case of homogenous agents. We begin with symmetric networks. Prices being independent of network structure in this context, outcome variations are exclusively related to demand effects. More precisely, the profit (resp. consumer surplus) is proportional to the sum (resp. to the sum of squares) of Bonacich centralities. We show that both profit and consumer surplus increase with information for all intensities of interaction, if and only if the network possesses the degree assortativity property. Degree assortativity has rarely been investigated in

\footnotetext{
${ }^{3}$ The notion of assortative mixing by individual characteristics such as age, gender, ethnicity, etc, is also called homophily - see Lazarsfeld and Merton [1954], McPherson, Smith-Lovin and Cook [2001].
} 
the field of network economics. To our knowledge, this paper is the first to link the value of network information with degree assortativity. Herein, we find that degree assortativity positively impacts the value of network information. Importantly, a well-known stylized fact is that social networks generally exhibit degree assortativity. ${ }^{4},{ }^{5}$

Turning to asymmetric networks, we observe that both the monopoly's profit and the consumer surplus depend on the network of averaged bilateral interactions. ${ }^{6}$ Introducing this latter network into the analysis, we establish that, when the network of averaged bilateral interactions is assortative by degree, network information induces an increase in both the monopoly's profit and the consumer surplus. This result is, once again, independent of the intensity of the interaction but, unlike the case of symmetric networks, degree assortativity is no longer a necessary condition.

We then enrich the study by incorporating agents' heterogeneity in the private preferences for the good. In this more general framework, it is not only degree assortativity that matters, but also the distribution of preferences on the network. The three following conditions are shown to guarantee that network information increases profit for all intensities of interaction: degree assortativity, homophily and a condition stating that the preference-degree assortativity coefficient should exceed a negative threshold; this threshold is equal to the opposite of the square root of the product of the degree assortativity coefficient by the coefficient of assortative mixing by preference (in particular, preference-degree assortativity fills this latter condition). Yet these conditions do not necessarily imply increased demand, which explains how consumer surplus can fall. We then show that, on top of these three previous conditions, preference-Bonacich centrality assortativity guarantees an increase in consumer surplus. We also identify a class of networks such that homophily alone guarantees that network information increases consumer surplus. This class is such that the average neighbors' degree is the same for all consumers. This class includes, but is not restricted to, regular networks, i.e., networks in which all agents have the same degrees.

We also discuss some limitations and extensions of the model, by examining two modifications of the information structure. We first consider the monopoly's profit when consumers know the distribution of in-degrees but have no information about out-degrees (they assume no correlation between in-degrees and out-degrees). Assuming homogenous preferences for simplic-

\footnotetext{
${ }^{4}$ See Newmann [2002], Table I, p. 2, or Serrano, Boguñá, Pastor-Satorras and Vespignani [2007].

${ }^{5}$ Interestingly, degree assortativity has become a hot topic in network analyses in physics, in biology and in the social sciences. For instance, degree assortativity is known to play an important role in diffusion processes and has an impact on connectivity properties of networks.

${ }^{6}$ The adjacency matrix of this network is the average between the adjacency matrix of the network and its transpose.
} 
ity, meeting two conditions guarantees that profit increases with information: in-degree assortativity, i.e. the tendency for consumers to be influenced by consumers of similar in-degrees, and positive correlation between in-degrees and out-degrees. Second, we check the robustness of our results to partial information: in the real world, firms and consumers may not know the full detail of the network due, for instance, to laws protecting privacy. Almost all results apply to the setting where agents know the number of links between each pair of types rather than the full network structure; again, key for the analysis is the equivalence, that we established before, between the game of incomplete information and the corresponding modified game of complete information.

Related literature. This paper fits into the classical IO literature on network effects initiated by Farrell and Saloner (1985) or Katz and Shapiro (1985). ${ }^{7}$ Recently, both Candogan et al (2012) and Bloch and Quérou (2013) study monopoly pricing under local network effects. In their setup agents are fully informed about the structure of the network of interaction. Fainmesser and Galeotti (2015) consider a model of incomplete network information. Consumers only know the distribution of in-degrees and out-degrees in the network. The authors study the impact of increased information for the monopoly on both the monopoly's profit and the welfare. ${ }^{8}$ Mainly, there are three differences with our setup. First, we compare situations in which consumers have distinct information sets. By doing so, we introduce the possibility of negative information value. Second, our work takes into account the network structure whereas theirs only considers degrees. ${ }^{9}$ We also introduce consumer heterogeneity.

This paper also fits into the literature on network games. Ballester, Calvò-Armengol and Zenou (2006), considering network effects in a game of linear-quadratic utilities under complete information, establish a relation between equilibrium play and Bonacich centrality. Jackson and Yariv (2005, 2007) study diffusion of behavior and equilibrium properties in a network game with incomplete information. Galeotti, Goyal, Jackson, Vega-Redondo and Yariv (2010) take this a step further, discussing strategic equilibrium in a wide set of network games. ${ }^{10}$ Considering utility functions concave in

\footnotetext{
${ }^{7}$ See also Economides (1996) for an extensive survey of this literature.

${ }^{8}$ In section 5 of their paper, Candogan et al (2012) consider both the case where the monopolist ignores network effects and the case of complete information, and examine the (positive) impact of network information to the monopolist.

${ }^{9}$ The degree distribution is not a comprehensive description of the network structure.

${ }^{10}$ Charness, Fery, Meléndez-Jiménez and Sutter (2014) perform some experimental economics and test network games with incomplete information. Other theoretical papers on network game with incomplete information are Sundararajan (2008), who studies local network effects and network structure in the context of the adoption of product innovation, and Kets (2011), in which agents cannot know the exact network size, nor can they know type correlations of their neighbors. More recently, Acemoglu, Malekian
} 
degrees, their paper compares outcomes from different degree distributions under positive local affiliation. Since we compare networks with the same joint degree distributions, first-order stochastic dominance is not an issue here. Furthermore, we reveal the impact of degree assortativity, which is less demanding than positive local affiliation.

The analysis of the impact of degree assortativity in economic outputs has so far received little attention. ${ }^{11}$ Rather, the literature examines network formation precesses generating degree assortativity. Jackson and Rogers (2007) propose a network formation process mixing random and networkbased searching. The dynamic aspect of the model produces assortativity, since older nodes are both more likely to be linked to each other and more likely to have a higher degree. Bramoullé, Currarini, Jackson, Pin and Rogers (2012) introduce individual heterogeneity to the framework of Jackson and Rogers (2007), and study how homophily affects network integration. König, Tessone and Zenou (2010) consider a dynamic model of network formation where agents form and sever links based on the centrality of their potential partners, and show by simulations that the existence of capacity constraints in the number of links an agent can maintain introduces a transition from disassortative to assortative networks. ${ }^{12}$

The notion of homophily is now widely studied in network economics; see for instance Currarini, Jackson and Pin (2009) for the formation of friendship networks, or Golub and Jackson (2012) in the context of learning. Recently, Galeotti and Rogers (2013) study the impact of (dis)assortative mixing in group characteristics on strategic immunization incentives. Cabrales, Gottardi and Vega-Redondo (2014) investigate the trade-off between the risksharing gains enjoyed by more interconnected firms and the costs resulting from increased risk exposure. They establish a link between the nature of shock distribution and optimal network segmentation, showing that when firms are heterogenous, optimality requires perfect homophily in a compo-

and Ozdaglar (2013) present a network game in which investment decisions are based on the realization of a random network formation process. It is also worth mentioning that, in network games, uncertainty may be related to other dimensions. See de Marti and Zenou (2015) for a model where the individual reward or the strength of interactions is only partially known by the agents and references therein.

${ }^{11}$ In an unpublished note, Feri and Pin (2016) study the impact of degree assortativity on the relationship between efforts and degrees in network games under incomplete information established in Jackson et al (2010).

${ }^{12} \mathrm{~A}$ current trend in physics is to study the formation of random networks, aiming to reproduce some stylized statistics inherent to real world networks (for a recent survey, see Pin and Rogers [2015]). Starting from the seminal model of Erdös and Rényi (1960), of special interest for economists are the so-called configuration models, which focus on random network formation with pre-determined degree distribution (see for instance Molloy and Reed [1995]). However, there has been recent emphazis on network formation models able to reproduce other statistics. Whereas Barabasi-Albert's preferential attachment random process generates a null coefficient of assortative mixing by degree for large populations (see Table I in Newman [2002]), recent models capture degree assortativity as an outcome of the network formation process (see for instance Hagberg and Lemmons [2015] and references therein). 
nent. Our paper, which combines heterogeneity with network effects, stresses the impact of both homophily, degree assortativity, and, originally, of the coefficients of preference-degree and preference-Bonacich centrality assortative mixing.

Our paper also echoes the literature studying the efficiency of stratification under complementarities. Becker (1973) studies the marriage market and mainly finds that assortative matching is efficient. Durlauf and Seshadri (2003) show that assortative matching is efficient if groups have the same size, and if agents' productivities are independent of the group composition. Bénabou (1996) discusses the short run versus long run impact of assortative matching on growth and inequalities. Our approach mainly departs from this literature by introducing a network structure between interacting agents.

The paper is organized as follows. In section 2, we present the model and characterize Nash equilibrium of both games of complete and incomplete information. In this section, we establish the relationship between the game of incomplete information and a modified game of complete information. We also introduce assortative mixing, and express assortativity coefficients as functions of the difference between the interaction matrices of the game of complete information and the modified game of complete information. Section 3 studies the sign of the value of information; we first present homogenous consumers' characteristics, and then introduce heterogeneity. Section 4 discusses the limitations and extensions of the results and section 5 concludes. The outcomes of the game of incomplete information are analyzed in Appendix A, and Appendix B gathers all the other proofs.

\section{The model}

We present a model in which a monopolist charges linear prices in the presence of local network effects among consumers. We will consider two situations: full information and incomplete information.

\subsection{The game}

We consider a two-stage game à la Candogan et al (2012). In the first period, the monopolist sets prices and in the second period, consumers buy a divisible quantity of the good. We let $x_{i} \in[0,+\infty)$ represent the quantity purchased by consumer $i$. The monopolist selects a vector $P=\left(p_{1}, p_{2}, \cdots, p_{n}\right)$ of prices where $p_{i} \geq 0$ represents the price charged to consumer $i$ for one unit of the good. The monopoly incurs a constant cost $c$ for each unit of the good produced. For convenience, we define $C=c \mathbf{1}$, where symbol $\mathbf{1}$ represents the 
$n$-dimensional vector of ones. In this context, the monopoly's profit is given by $\sum_{i}\left(p_{i}-c\right) x_{i}$.

There is a set $N=\{1,2, \cdots, n\}$ of consumers organized in a social network. The $n \times n$ matrix $G=\left[g_{i j}\right]$, with $g_{i j} \in\{0,1\}$, represents the network of interaction between consumers (we will refer to it as network $G$ ). We let $g_{i j}=1$ whenever agent $i$ is influenced by agent $j .{ }^{13}$ By convention, $g_{i i}=0$ for all $i$. We let $I$ denote the $n$-dimensional identity matrix. We define the profiles $K=G \mathbf{1}, L=G^{T} \mathbf{1}$, where the respective entries $k_{i}, l_{i}$ denote agent $i$ 's in-degree and out-degree in network $G$, we let $g=\mathbf{1}^{T} G \mathbf{1}$ be the sum of in-degrees (or out-degrees) in network $G, d=\frac{g}{n}$ denote the average in-degree.

We let parameter $a_{i}$ represent consumer $i$ 's private preference for the good, and we let $A=\left(a_{1}, a_{2}, \cdots, a_{n}\right)$ represent the profile of preferences. The utility that agent $i$ derives from consuming quantity $x_{i}$ of the good is given by:

$$
u\left(x_{i}, x_{-i}\right)=a_{i} x_{i}-\frac{1}{2} x_{i}^{2}+\delta \sum_{j \in N} g_{i j} x_{i} x_{j}-p_{i} x_{i}
$$

The present utility specification, introduced by Ballester et al (2006), presents the following features. First, there is an idiosyncratic component composed of private preference for the good, and a satiety effect (or usage cost) which fixes some finite optimal consumption level even under zero price. Second, there are peer effects. Peer consumption levels entail local positive externalities: consumers' utilities increase with neighbors' consumption levels. Moreover, there are local complementarities: the higher the consumption level of neighbors, the greater the incentive for agent $i$ to increase his consumption level.

Under complete information, consumers and the monopoly are fully informed about network structure and preferences. Now we turn to the game of incomplete network information. Each consumer knows the joint distribution of in-degrees, out-degrees and preferences, as well as her own private preference, in-degree and out-degree. In this situation, consumers are naturally typed by the triplet $(a, k, l)$. We define by $\mathcal{T}=\{(a, k, l)\}$ the set of consumer types, and and we let characteristics $\left(a_{t}, k_{t}, l_{t}\right)$ corresponds to type $t$.

The monopoly knows every consumer's type, as well as the joint distribution of types. We let $s_{t}$ represent the number of consumers of type $t \in \mathcal{T}$

\footnotetext{
${ }^{13}$ In the real world, the nature of interactions can differ substantially according to the economic context. For instance, for communication goods such as games, mobiles devices and phones, computers, bilateral interactions between consumers are essentially symmetric. Conversely, for experience goods like cultural goods, or for fashion goods, bilateral influence may not be symmetric. While my friend's view strongly influences my likelihood of purchasing a book or seeing a movie, it may be that my own view does not influence this friend. Our setting encompasses all these types of interactions.
} 
in network $G$. We assume that consumers of the same type $t$ choose the same consumption level, generically called $x_{t}$ (this assumption is standard in the literature, see Jackson et al [2010]). Following Fainmesser and Galeotti (2015), the expected utility of consumer $i$ is given by

$$
E U_{i}\left(x_{i}, x_{-i}\right)=\left(a_{i}-p_{i}\right) x_{i}-\frac{x_{i}^{2}}{2}+\delta x_{i} k_{i} A V(X)
$$

where $A V(X)$ refers to the average consumption of neighbors. In order to compute the probability of a neighbor's type to be $t$, consumers should consider all networks with the same joint distribution of types as network $G$. Importantly, they do not take into account correlation between types of linked consumers. We also assume that the joint distribution of types does not provide further information on the network structure. ${ }^{14}$ When the network is sufficiently large, this probability can be approximated by $l_{t} s_{t} / g$. This result is common in the literature on incomplete information on networks (see for instance Jackson et al [2010], or recently Fainmesser and Galeotti [2015]). The expected average consumption level of neighbors is thus equal to $\sum_{t \in \mathcal{T}} \frac{l_{t} s_{t}}{g} x_{t}$. Now, we observe that

$$
\sum_{t \in \mathcal{T}} l_{t} s_{t} x_{t}=\sum_{j \in N} l_{j} x_{j}
$$

Defining matrix $H=\frac{K L^{T}}{g}$ (so $h_{i j}=\frac{k_{i} l_{j}}{g}$ for all $i, j$ ), consumer $i$ 's expected utility is written:

$$
u_{i}\left(x_{i}, x_{-i}\right)=\left(a_{i}-p_{i}\right) x_{i}-\frac{x_{i}^{2}}{2}+\delta x_{i} \sum_{j \in N} h_{i j} x_{j}
$$

Hence, the Bayesian Nash equilibrium of the second stage of the incomplete information game played on network $G$ generates the same consumption profile as the Nash equilibrium of the (virtual) game with complete information played on network $H$, where the impact of consumer $j$ on consumer $i$ 's expected utility depends on agent $i$ 's in-degree $k_{i}$ and agent $j$ 's out-degree $l_{j}$. Note that both $G$ and $H$ have the same in-degree distribution.

Consider a non-negative matrix $W$. We let $\mu(W)$ denote the largest eigenvalue of matrix $W$. We impose the following assumptions throughout the paper:

Assumption 1. $\delta<\frac{1}{\max \left(\mu\left(\frac{G+G^{T}}{2}\right), \mu\left(\frac{H+H^{T}}{2}\right)\right)}$

\footnotetext{
${ }^{14}$ For instance, the degree distribution of a star network reveals the full network structure.
} 
Assumption 2. $c<\min \left\{a_{1}, a_{2}, \cdots, a_{n}\right\}$

Together, assumptions 1 and 2 guarantee that both games admit unique and interior solutions: the latter guarantees a positive equilibrium demand, the former guarantees that optimal consumption levels are finite.

For a given price vector, the first-order condition on the demand of consumer $i$ on network $W$ is written:

$$
x_{i}^{B R}=a_{i}-p_{i}+\delta \sum_{j \in N} w_{i j} x_{j}
$$

The optimal profit and consumer surplus can therefore be expressed as functions of the position of agents on the network through centrality indexes defined as follows. We let the profile $B(W, \delta)=(I-\delta W)^{-1} \mathbf{1}$ represent the Bonacich centrality of network $W$ under decay parameter $\delta$. The quantity $b_{i}(W, \delta)$ represents the number of weighted paths from agent $i$ to others, where the weight of a path of length $k$ from agent $i$ to agent $j$ is $\delta^{k}$. The profile $B_{Z}(W, \delta)=(I-\delta W)^{-1} Z$ represents the Bonacich centrality of network $W$ weighted by vector $Z$. Assumption 1 guarantees $(I-\delta W)^{-1}>0$. For convenience, we will omit reference to parameter $\delta$ in centralities, profit and consumer surplus. We also introduce the euclidian vectorial norm $\|Z\|=$ $\sqrt{Z^{T} Z}$. We recall:

Proposition 1. When consumers interact on network $W$, the optimal profit of the monopoly is written

$$
\Pi(W)=\frac{1}{4}(A-C)^{T} B_{A-C}\left(\frac{W+W^{T}}{2}\right)
$$

and the consumer surplus is written

$$
C S(W)=\frac{1}{8}\left\|B_{A-C}\left(\frac{W+W^{T}}{2}\right)\right\|^{2}
$$

Moreover, prices are written

$$
P(W)=A-\frac{1}{2}(I-\delta W)\left(I-\delta \frac{W+W^{T}}{2}\right)^{-1}(A-C)
$$

and consumption levels are equal to

$$
X(W)=\frac{1}{2}\left(I-\delta \frac{W+W^{T}}{2}\right)^{-1}(A-C)
$$


Thus, for network $W$, both the profit and the consumer surplus depend on the network $\frac{W+W^{T}}{2}$. Moreover, when $W=W^{T}$, the price vector $P(W)=\frac{A+C}{2}$ is independent of the network structure.

For the incomplete information game, we can obtain outcomes as functions of the statistics of the distribution of types (see Appendix A). Comparing these expressions to the outcomes of the complete information game is tricky. Expressing equilibrium outcomes as Bonacich centralities proves key to comparing the monopoly's profit and the consumer surplus in the games of complete and incomplete information.

\subsection{Assortative mixing}

Newman (2003, eq. 21) defines a coefficient of assortative mixing by a characteristic, which measures the tendency for agents to be linked to agents with similar characteristics. Here we generalize this coefficient to two scalar characteristics.

We consider two scalar characteristics of respective supports $\mathcal{C}_{1}, \mathcal{C}_{2}$, and two vectors $Z \in \mathcal{C}_{1}^{N}, Z^{\prime} \in \mathcal{C}_{2}^{N}$. We define assortative mixing between characteristics $Z$ and $Z^{\prime}$ on network $G$ as follows:

$$
r_{z, Z^{\prime}}(G)=\frac{\sum_{z \in \mathcal{C}_{1}, z^{\prime} \in \mathcal{C}_{2}} z z^{\prime}\left(e_{z z^{\prime}}-a_{z} b_{z^{\prime}}\right)}{\sigma_{a} \sigma_{b}}
$$

where $e_{z z^{\prime}}$ is the fraction of all edges in network $G$ that join together vertices of characteristics $z$ and $z^{\prime}, a_{z}$ represents the fraction of edges that start with characteristics $z, b_{z^{\prime}}$ represents the fraction of edges that end with characteristics $z^{\prime}, \sigma_{a}$ and $\sigma_{b}$ the standard deviations of the distributions $a_{z}$ and $b_{z^{\prime}}$. Note that if there is no assortative mixing between the characteristics of two linked agents, the fraction $e_{z z^{\prime}}$ should be equal to $a_{z} b_{z^{\prime}}$. We say that a network has the property of assortativity (resp. disassortativity) between characteristics $Z$ and $Z^{\prime}$ whenever $r_{Z, Z^{\prime}}(G)>0$ (resp. $\left.<0\right)$.

We present now an alternative expression of the coefficient $r_{Z, Z^{\prime}}(G)$, which proves crucial to the analysis:

Proposition 2. Consider network $G$, let $H=\frac{K L^{T}}{g}$ and let $\Theta=G-H$. For any pair of scalar characteristics $Z, Z^{\prime}$, we have

$$
r_{Z, Z^{\prime}}(G)=\frac{1}{g \sigma_{a} \sigma_{b}} Z^{T} \Theta Z^{\prime}
$$

Assortative mixing by a single characteristic corresponds to the case where $Z=Z^{\prime}$, and we call $r_{Z}(G)=r_{Z, Z}(G)$ for convenience. Four coefficients, all of which apply to symmetric network, will play a key role in the paper: 
- The first coefficient is $r_{D}(G)$ (where $D=G \mathbf{1}$ represents the profile of individual degrees). Degree assortativity holds whenever $r_{D}(G)>0$, which indicates that consumers are more likely to be linked to consumers with similar degrees. ${ }^{15}$ By Proposition 2, we have $r_{D}(G)>0$ whenever $D^{T} \Theta D>$ 0 . This means that the number of paths of length 3 in network $G$ exceeds the number of weighted paths of length 3 in network $H .{ }^{16}$ Moreover, assortative mixing by degree can be characterized in terms of paths in network $G$ only. Letting $g^{(k)}=\mathbf{1}^{T} G^{k} \mathbf{1}$ represent the number of paths of length $k \geq 1$ in network $G$, we get

$$
r_{D}(G)>0 \text { if and only if } g^{(1)} g^{(3)}>\left(g^{(2)}\right)^{2}
$$

i.e., the product of twice the number of links by the number of paths of length 3 exceeds the square of the number of paths of length 2 .

- The second coefficient, $r_{A}(G)$, measures the level of (dis)assortative mixing by characteristic $A$. Homophily then refers to the case in which $r_{A}(G)>0$ (i.e., $A^{T} \Theta A>0$ ), and indicates that consumers are more likely to be linked to consumers with similar preferences.

- The third coefficient is $r_{A, D}(G)$. It measures the tendency of highpreference consumers to be linked to high-degree consumers. When $r_{A, D}(G)>$ 0 (i.e., $A^{T} \Theta D>0$ ), there is preference-degree assortativity.

- The fourth coefficient is $r_{A, B(G)}(G)$. It measures the tendency of highpreference consumers to be linked to high-Bonacich centrality consumers. When $r_{A, B(G)}(G)>0$ (i.e., $A^{T} \Theta B(G)>0$ ), there is preference-Bonacich centrality assortativity.

\section{The value of network information}

In this section, we compare both the monopoly's profit and the consumer surplus in the game with complete network information and the game under incomplete network information. Generally speaking, under incomplete information, consumers do not know the true type of their neighbors, simply inferring expected neighbors' consumption levels from their knowledge about the distribution of types. Once agents obtain information about the structure of the network of interaction, consumers modify their consumption levels and the monopoly may change prices. This results in a variation in both the profit and the consumer surplus.

\footnotetext{
${ }^{15}$ As extreme cases, the Pearson coefficient of a complete bipartite network, including the star network, takes the value -1 (however, in general non-complete bipartite networks can be assortative). By contrast, the Pearson coefficient of the union of two regular components with at least two distinct degrees takes the value 1.

${ }^{16}$ We did not identify any such characterization of degree assortativity in the literature.
} 
To understand the role of the network structure, we start with the benchmark case of homogenous consumers. Then we incorporate heterogeneity.

\subsection{Homogenous preferences}

In this subsection, we consider homogenous preferences and we set $A=\mathbf{1}$. In this setting, the monopoly's profit is proportional to the sum of Bonacich centralities, and consumer surplus is proportional to the sum of squares of centralities. To determine the sign of the value of network information, we have to compare Bonacich centralities corresponding to the games played on networks $G$ and $H$, both of which have the same degree distribution. In general, both positive and negative information values are possible outcomes.

We start by examining the case of symmetric networks, i.e. we assume $G^{T}=G$. We note that prices are unchanged since network $G$ is symmetric, meaning that outcome gaps are the result of a pure demand effect. As a first observation, it is easily seen that the value of information is null on regular networks (where all agents have the same degree), because centralities are homogenous and identical in both networks.

Figure 3.1 depicts two networks with the same number of consumers, the same degree distribution, and with two classes of consumers in terms of structural positioning (in the figure, black nodes represents consumers with degree 3 , white nodes consumers with degree 2). Vector $X$ (resp. $Y$ ) is the consumption profile in the game of complete (resp. incomplete) information. For $\delta=0.2$, network $G_{1}$ represented in Figure 3.1-Left (resp. $G_{2}$ in Figure 3.1-Right) generates a negative (resp. positive) profit gap. In network $G_{1}$ (resp. $G_{2}$ ), consumers with the highest degrees decrease (resp. increase) their consumption level with information, while consumers with the lowest degrees increase (resp. decrease) their consumption level. In total, in network $G_{1}$ (resp. network $G_{2}$ ) information generates a decrease (resp. increase) in aggregate consumption and profit. It can be seen that in network $G_{1}$, highdegree consumers tend to be linked with low-degree consumers, while in network $G_{2}$ consumers with equal degrees are exclusively linked with each other. The example suggests that degree assortativity matters.

Actually, when there is degree assortativity, central consumers tend to underestimate their neighbors' consumption levels under incomplete information (while peripheral consumers tend to overestimate their neighbors' consumption levels). Therefore, by complementarities, central consumers increase their consumption level with information, while peripheral consumers decrease their consumption level. However, it is unclear which effect dominates, although the example suggests that information is beneficial for the network satisfying degree assortativity. 


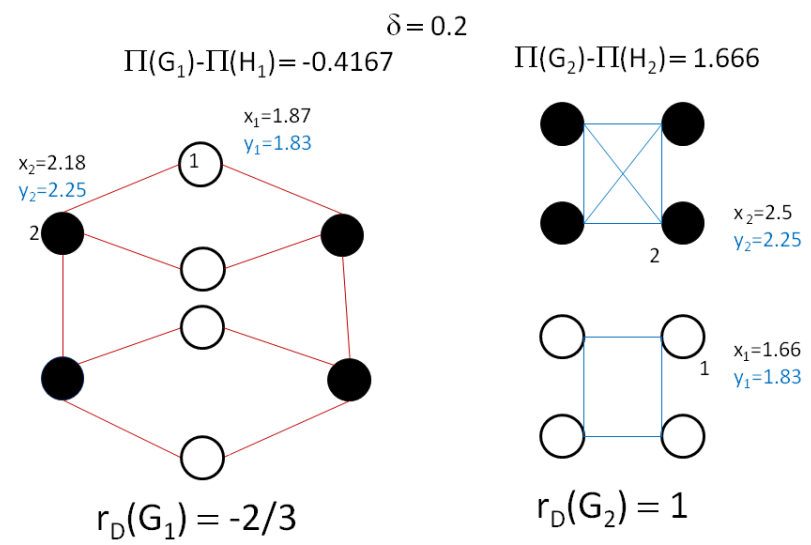

Figure 1: Two networks with the same number of consumers and the same degree distributions. Black consumers have degree 3, white consumers have degree 2. Left (resp. Right) is disassortative (resp. assortative) by degree.

We will see that, indeed, the sign of $r_{D}(G)$ is crucial in determining the sign of the profit gap. To grasp an intuition, we begin with the case where the intensity of interaction is close to zero. Recall that in order to assess the variation in consumption we need to compare aggregate Bonacich centralities in the two networks $G$ and $H$. Given that the numbers of paths of length 1 and 2 in networks $G$ and $H$ are identical, the sign of the gap in aggregate centralities is the same as the sign of the difference between the numbers of paths of length 3 in networks $G$ and $H$ when the intensity of interaction is sufficiently low. Now, as explained above, this difference is precisely equal to $D^{T} \Theta D .{ }^{17}$ Therefore, for a symmetric network $G$, when the intensity of interaction is sufficiently low, we have both $\Pi(G)>\Pi(H)$ and $C S(G)>$ $C S(H)$ if $r_{D}(G)>0$.

Does this result hold for higher intensities of interaction? Paths of lengths greater than 3 can no longer be ignored. But degree assortativity is still relevant:

Theorem 1. Consider a network $G$.

When $\Theta D \neq \boldsymbol{O}$, we have both $\Pi(G)>\Pi(H)$ and $C S(G)>C S(H)$ for all $\delta \in] 0, \frac{1}{\mu(G)}\left[\right.$ if and only if $r_{D}(G) \geq 0$.

When $\Theta D=\boldsymbol{O}$, we have both $\Pi(G)=\Pi(H)$ and $C S(G)=C S(H)$ for all $\delta \in] 0, \frac{1}{\mu(G)}[$.

\footnotetext{
${ }^{17}$ Actually, $\mathbf{1}^{T}\left(G^{3}-H^{3}\right) \mathbf{1}=D^{T} \Theta D$ because $H \mathbf{1}=G \mathbf{1}$.
} 
Theorem 1 is powerful because the assortativity condition is independent of the intensity of interaction. Moreover, it is worth mentioning that it is not possible to obtain a finer condition that is independent of the intensity of interaction, because the sufficient condition becomes necessary too when the intensity of interaction is sufficiently low.

For the class of networks satisfying $\Theta D=\mathbf{0}$, the two games have the same outcomes, and even the same consumption levels, so this class corresponds to the degenerate situation in which network information does not affect behaviors. A network satisfies $\Theta D=\mathbf{0}$ if the average neighbors' degree is the same for all consumers. Moreover, there is no assortative mixing by degree in these networks. This class of networks includes regular networks, but also some other network structures, as illustrated by the twelve-consumer sixteen-link network depicted in figure 3.1:

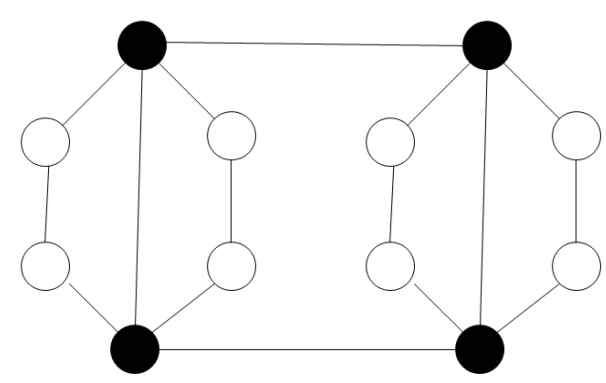

Figure 2: A non-regular network such that $\Theta D=\mathbf{0}$. Black consumers have degree 4 , white have degree 2.

Theorem 1 is silent about networks such that $r_{D}(G)<0$. For sufficiently small intensity of interaction, degree disassortativity involves a negative information value. But the sign of the information value on the same network differs according to the intensity of interaction. Generally speaking, for high intensity of interaction, a disassortative network can generate a positive outcome gap. Let $\lambda_{\min }(G)$ represent the minimum eigenvalue of network $G$. We obtain:

Proposition 3. Consider a network such that $\Theta D \neq \boldsymbol{O}$. We have both 


$$
\begin{aligned}
& \Pi(G)>\Pi(H) \text { and } C S(G)>C S(H) \text { if } r_{D}(G) \geq r_{c} \text { with } \\
& \qquad r_{c}=-\left(\frac{\delta \nu^{2}}{1-\delta \lambda_{\min }(G)}\right)\left(\frac{g^{2} g^{(4)}+\left(g^{(2)}\right)^{3}-2 g g^{(2)} g^{(3)}}{g \sum_{i} d_{i}^{3}-\left(g^{(2)}\right)^{2}}\right)
\end{aligned}
$$

We also examine how the profit gap varies with parameter $\delta$. This comparative statics analysis yields an unambiguous conclusion under homogenous preferences:

Corollary 1. Consider a network such that $\Theta D \neq \boldsymbol{O}$. When $r_{D}(G) \geq 0$, the profit gap is increasing in parameter $\delta$.

Hence, for all network structures, the higher the intensity of interaction between consumers, the higher the profit gap.

We turn to the general case of asymmetric networks. We introduce the intermediary network $\tilde{G}=\frac{G+G^{T}}{2}$ of averaged interactions. For convenience, we define $\tilde{D}=\frac{K+L}{2}$. We let matrix $\tilde{H}=\frac{\tilde{D} \tilde{D}^{T}}{g}$ denote the matrix of interaction of the game with incomplete information on network $\tilde{G}$. First, we observe that, under complete network information, outcomes are identical on networks $G$ and $\tilde{G}$. Second, we show that, for all intensities of interaction, outcomes are larger on the game played on network $\tilde{H}$ than the game played on network $H$. Elaborating on these two points, the next theorem emphasizes the role played by degree assortativity on the network of averaged interactions:

Theorem 2. Assume $(\tilde{G}-\tilde{H}) \tilde{D} \neq 0$. Then $\Pi(G)>\Pi(H)$ and $C S(G)>$ $C S(H)$ for all $\delta \in] 0, \frac{1}{\mu(G)}\left[\right.$ if $r_{\tilde{D}}(\tilde{G}) \geq 0$ (i.e., $\tilde{D}^{T}(\tilde{G}-\tilde{H}) \tilde{D} \geq 0$ ).

Theorem 2 generalizes Theorem 1 to non-symmetric networks. However, it is important to point out that, because we introduce the intermediary network $\frac{G+G^{T}}{2}$, the condition is no longer necessary, in constrast with the case of symmetric networks.

\subsection{Heterogenous preferences}

In this section, we take into account heterogenous private preferences for the good. This adds complexity to the model, because now agents may be distinct both in their position on the network and in their private preference. We assume here $G^{T}=G$, for simplicity. To get some intuition, we begin with the two polar cases of high and low intensities of interaction.

We start with a low intensity of interaction. Here, network effects are mainly driven by preference parameters. The following proposition shows the crucial role played by homophily: 
Proposition 4. Assume that $\delta$ is sufficiently small. We have both $\Pi(G)>$ $\Pi(H)$ and $C S(G)>C S(H)$ if $r_{A}(G)>0$.

We turn to the case where the intensity of interaction is sufficiently high. We have shown that the condition $r_{D}(G)>0$ guarantees that the sum of Bonacich centralities is strictly larger on network $G$ than on network $H$ for all intensities of interaction. Moreover, for network $G$ (resp. $H$ ), Bonacich centralities tend to infinity when $\delta$ tends to $\frac{1}{\mu(G)}$ (resp. $\left.\frac{1}{\mu(H)}\right)$. Thus, we have $\mu(G) \geq \mu(H)$. A direct consequence is that, when parameter $\delta$ tends to $\frac{1}{\mu(G)}$, both profit and consumer surplus tend to infinity on network $G$, but not on network $H$, irrespective of the distribution of vector $A$ on the network. We therefore obtain the following proposition:

Proposition 5. When $\delta$ tends to $\frac{1}{\mu(G)}$, we have both $\Pi(G)>\Pi(H)$ and $C S(G)>C S(H)$ if $r_{D}(G)>0$.

Now, we consider an arbitrary intensity of interaction. We first consider profit. A legitimate question is whether having both degree assortativity and homophily guarantees that profit increases with information. The answer is, it doesn't: when high-preference consumers are rather connected to lowdegree consumers, there can be a negative information value, as illustrated in example 1:

Example 1. In this example, we have both $r_{A}(G)>0, r_{D}(G)>0$ but $\Pi(G)-$ $\Pi(H)<0$.

We set $n=5, \delta=0.2, c=0$, and we consider the network and preferences depicted in Figure 1. We find $r_{A}(G) \simeq 0.06, r_{D}(G)=1, \Pi(G)-\Pi(H)=$ -0.1997 .

The next theorem shows that the coefficient of assortative mixing between preferences and degrees does matter:

Theorem 3. Consider a network $G$.

When $\Theta A \neq \boldsymbol{O}$ or $\Theta D \neq \boldsymbol{O}$, we have $\Pi(G)>\Pi(H)$ for all $\delta \in] 0, \frac{1}{\mu(G)}[$ if $r_{D}(G) \geq 0, r_{A}(G) \geq 0$ and $r_{A, D}(G) \geq-\sqrt{r_{A}(G) \cdot r_{D}(G)}$.

When $\Theta A=\Theta D=\boldsymbol{O}$, we have $\Pi(G)=\Pi(H)$ for all $\delta \in] 0, \frac{1}{\mu(G)}[$.

In short, the three assortative mixing conditions ${ }^{18}$ guarantee that degrees and preferences reinforce each other in such a way that the average increase in the consumption level of central consumers dominates the decrease of less

\footnotetext{
${ }^{18}$ Note that the three conditions $r_{D}(G)>0, r_{A}(G)>0$ and $r_{A, D}(G)>0$ do not involve a positive correlation between preferences and degrees.
} 


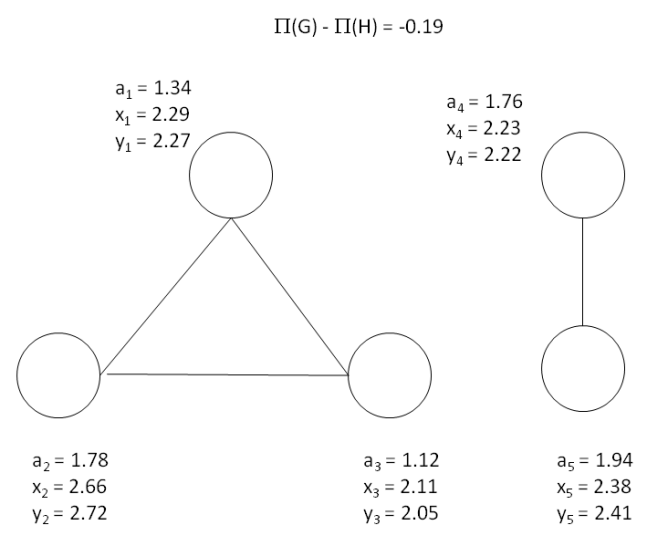

Figure 3: The network, preferences and consumption levels of the two games in example 1.

central consumers. Conversely, when the disassortativity between preferences and degrees is too strong, preferences and degrees are misaligned, which can produce a decrease in profit.

To sum up, under low intensity of interaction, homophily $\left(r_{A}(G)>0\right)$ guarantees that profit increases with information. Under very high intensity of interaction, degree assortativity $\left(r_{D}(G)>0\right)$ guarantees a positive information value. For intermediate intensity of interaction, not only do these two conditions matter, but in addition, preferences and degrees need to be not too disassortatively mixed (of course, preference-degree assortativity fills this latter condition).

We turn now to consumer surplus. Actually, the three conditions of Theorem 3 do not guarantee an increase in consumer surplus. Example 2 illustrates the point:

Example 2. In this example, we have $r_{A}(G)>0, r_{D}(G)>0, r_{A, D}(G)>$ $-\sqrt{r_{A}(G) r_{D}(G)}$ but $C S(G)-C S(H)<0$.

Consider $n=6, \delta=0.1624, c=0$, and consider the network and preferences depicted in Figure 2. Then, we have $r_{A}(G)=0.228, r_{D}(G)=0.333$, $r_{A, D}(G)=-0.258>-\sqrt{r_{A}(G) \cdot r_{D}(G)}=-0.275$ and $C S(G)-C S(H)=$ -0.0087 . We also have $\Pi(G)-\Pi(H)=0.1190$ and $\mathbf{1}^{T}(X-Y)=-0.0119$.

In the above 6-consumer example, aggregate demand decreases with network information because high-preference consumers are rather linked to 


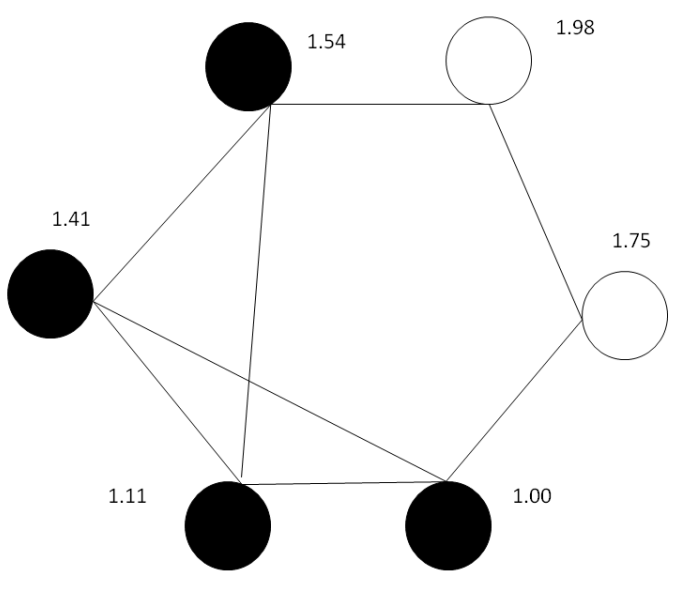

Figure 4: The network and preferences in example 2. Black nodes have degree 3 , and white have degree 2 .

low-degree consumers. This in turn originates a decrease in the consumer surplus.

We give now conditions under which network information increases the consumer surplus. Recall that $r_{A, B(G)}(G)$ measures assortative mixing between Bonacich centralities and preferences. We obtain:

Theorem 4. Consider a symmetric network $G$.

When $\Theta A \neq \boldsymbol{O}$ or $\Theta D \neq \boldsymbol{O}$, we have $C S(G)>C S(H)$ if the following conditions hold: $r_{A}(G) \geq 0, r_{D}(G) \geq 0, r_{A, D}(G) \geq-\sqrt{r_{A}(G) \cdot r_{D}(G)}$, and $r_{A, B(G)}(G) \geq 0$.

When $\Theta A=\Theta D=\boldsymbol{O}$, we have both $C S(G)=C S(H)$ for all $\delta \in] 0, \frac{1}{\mu(G)}[$.

Key in Theorem 4 is that preference-Bonacich centrality assortativity guarantees increased demand, which in turn, combined with increased profit, garantees an increase in the consumer surplus.

It should be stressed that the condition $r_{A, B(G)}(G) \geq 0$ depends on the intensity of interaction. This means that, as parameter $\delta$ varies, the sign of the coefficient $r_{A, B(G)}(G)$ can change. We explored whether the three conditions $r_{A}(G) \geq 0, r_{D}(G) \geq 0, r_{A, D}(G) \geq 0$ guarantee an increase in consumer surplus (for networks such that either $\Theta A \neq \mathbf{0}$ or $\Theta D \neq \mathbf{0}$ ). They do, under both sufficiently low and sufficiently high intensities of interaction. However, for intermediate intensities, the question remains open. One difficulty is that demand can decrease under these three conditions, as shown by example 3 : 
Example 3. In this example, we have both $r_{A}(G)>0, r_{D}(G)>0, r_{A, D}(G)>$ 0 but $\mathbf{1}^{T}(X-Y)<0$.

Consider $n=9, \delta=0.05, c=0$, and consider the network and preferences depicted in Figure 3. We find $r_{A}(G) \simeq 0.0196, r_{A, D}(G) \simeq 0.0087, r_{D}(G) \simeq$

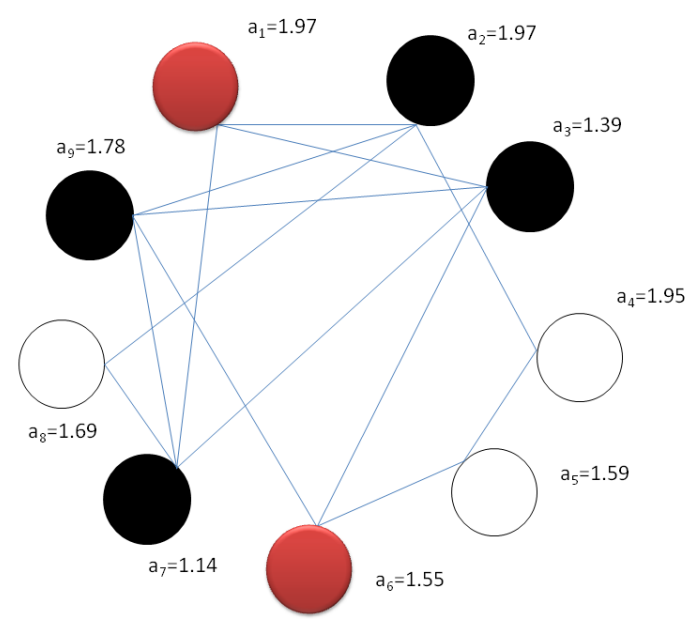

Figure 5: The network and preferences in example 3 - Black nodes have degree 4 , red have degree 3 , and white have degree 2 .

0.0233 , and $\mathbf{1}^{T}(X-Y) \simeq-1.18 e-04$. Furthermore, we have $C S(G)-$ $C S(H)=0.0048$.

Remark: networks satisfying $\Theta D=\mathbf{0}$ and $\Theta A \neq \mathbf{0}$. When the network satisfies $\Theta D=\mathbf{0}$ (e.g., regular networks), we have $r_{D}(G)=r_{A, D}(G)=$ $r_{A, B(G)}(G)=0$. Proposition 4 and Theorem 3 then show that, when $\Theta A \neq \mathbf{0}$, homophily guarantees a positive network information value. We note that the sum of consumption levels is identical in the two games. ${ }^{19}$

Remark: ex post consumer surplus. The consumer surplus describes the sum of equilibrium ex ante utilities, i.e. utilities derived from consumption levels $Y$ on expected network $H$. Ex post utilities, say $C S^{\text {ex }}{ }^{\text {post }}$, derived from consumption levels $Y$ on real network $G$, also deserve attention. We can easily show that $C S^{\text {ex post }}=C S(H)+\delta Y^{T} \Theta Y$. Therefore, the conditions guaranteeing a positive profit gap, as given in Theorem 3, also involve that $C S^{\text {ex post }}>C S(H)$. We can also show that under the conditions given in Theorem 4, $C S(G)-C S(H)>\delta Y^{T} \Theta Y$ (see equation (24)), which implies that $C S(G)>C S^{\text {ex }}$ post.

\footnotetext{
${ }^{19}$ Since un-weighted centralities are homogenous on regular networks, $\Theta M \mathbf{1}=\mathbf{0}$, and thus $\mathbf{1}^{T}(X-Y)=$ $\delta(M \mathbf{1})^{T} \Theta Y=0$.
} 


\section{Discussion}

In this section, we discuss two modifications of the information structure. We first compare the game with complete information and the game in which consumers only know the in-degree distribution. Second, we examine partial information: we assume that agents know the number of links between each pair of types, instead of the full information about the game.

Knowing in-degrees. We examine the situation in which consumers only know their own in-degree and the in-degree distribution of the network. Agents assume that there is no correlation between in-degrees and outdegrees. To simplify the analysis, we assume that consumers are homogenous.

As in the above cases, we build a matrix of interaction by agent for the game of incomplete information. Since each consumer considers all consumers in the society as potential partners with equal probabilities, the matrix of interaction becomes $\bar{H}=\frac{K \mathbf{1}^{T}}{n}$ (so, $\bar{h}_{i j}=\frac{k_{i}}{n}$ for all $i, j$, including the diagonal).

We consider the general case where network $G$ can be asymmetric. The tendency of consumers to be influenced by consumers of similar in-degrees affects the value of information. We call $r_{K}(G)$ the coefficient of in-degree assortativity. We also let $\rho_{k, l}$ represent the correlation between in-degrees and out-degrees in network $G$. We obtain:

Proposition 6. We have $\Pi(G) \geq \Pi(\bar{H})$ if both $r_{K}(G)>0$ and $\rho_{k, l} \geq 0$.

Proposition 6 shows that in-degree assortativity, combined with a positive correlation between in-degrees and out-degrees, guarantees a positive profit gap. Essentially, positive correlation guarantees high transmission of influence on the network, because big influencees are also more likely to be big influencers. Note that if in-degrees and out-degrees are independent, only in-degree assortativity matters.

Partial information. In many circumstances, the monopolist and consumers do not know the full detail of the network, for instance due to laws protecting privacy. ${ }^{20}$ Here we relax the assumption of full information, assuming instead that agents only know the number of links between each pair of types (and, as before, the monopoly observes each consumer characteristic). To fix ideas, we assume a symmetric network $G$. We let $\psi_{t t^{\prime}}$ represent the total number of links between pairs of types $t$ and $t^{\prime}$ (where a type refers

\footnotetext{
${ }^{20}$ Current legislation regarding the protection of privacy on Internet markedly differs across nations, and is under constant debate. For instance, legal protection against private institutions is rather weak in the US (outside the Children's Online Privacy Protection Act), while it is stronger in the EU; e.g., European Convention number 108 for the Protection of Individuals with regard to Automatic Processing of Personal Data, or the role of the Article 29 Data Protection Working Party.
} 
to a pair $(a, d))$. Then, if consumer $i$ is of type $t$ and consumer $j$ is of type $t^{\prime}$, we can show that the corresponding matrix of interaction by agent is $Q$ where $q_{i j}=\frac{\psi_{t t^{\prime}}}{s_{t} s_{t^{\prime}}}$, with $s_{t}$ being the number of consumers of type $t .{ }^{21}$ Then, matrix $Q$ has the same degree distribution and assortativity coefficients as network $G$, i.e. $r_{A}(G)=r_{A}(Q), r_{D}(G)=r_{D}(Q), r_{A, D}(G)=r_{A, D}(Q)$, so all corresponding results stay valid.

Partial information allows for a second interpretation of our results, where the network is formed after consumer decisions (as in Jackson et al [2010]), Acemoglu et al [2013] or Fainmesser and Galeotti [2015]). In the first situation, agents only know the joint distribution of types and assume no correlation between types of linked agents; in the second situation, they know both the joint distribution of types and public information about the process of network formation summarized by the matrix $P=\left[p_{t t^{\prime}}\right]$, where $p_{t t^{\prime}}$ represents the probability that a neighbor of an agent of type $t$ is of type $t^{\prime}$. The corresponding interaction matrix by agent is therefore given by matrix $Q$, where $q_{i j}=\frac{d_{t} p_{t t^{\prime}}}{s_{t^{\prime}}}{ }^{22}$ We can therefore apply our results to compare outcomes of the games played on networks $Q$ and $H$. Note that the consumption profile of the game played on network $Q$ is a Bonacich centrality and can no longer be characterized by simple network statistics.

\section{Conclusion}

In this paper we considered monopoly pricing in a context where consumers are organized in a network of local complementarities. We explored whether network information is valuable to firms and consumers. Our analysis yields clear-cut results on how network structure affects the value of network information, showing the positive impact of degree assortativity, homophily, preference-degree assortativity and preference-Bonacich centrality assortativity. These results are interesting in the light of the empirically documented properties of social networks: degree assortativity and homophily.

Several questions related to this paper remain open. First, competition among firms deserves attention. For instance, it could be valuable to explore how the fierceness of competition affects the value of information in the presence of network effects. ${ }^{23}$ Second, network evolution is an issue. Firms

\footnotetext{
${ }^{21}$ For consumer $i$ with degree $d_{i}$, the probability that the type of a neighbor is $t^{\prime}$ is given by $\frac{\psi_{t t^{\prime}}}{d_{i} s}$. In order to obtain the system of interaction by agent, we multiply this quantity by $d_{i}$ and divide by $s_{t^{\prime}}$, the number of agents of type $t^{\prime}$.

${ }^{22}$ Note that $q_{i j}=\frac{d_{t} s_{t} p_{t t^{\prime}}}{s_{t} s_{t^{\prime}}}$ and $d_{t} s_{t} p_{t t^{\prime}}$ represents the expected number of links between types $t$ and $t^{\prime}$. Therefore, matrix $Q$ is symmetric.

${ }^{23}$ For multiproduct oligopolies, Chen, Zenou and Zhou (2015) show that, in the presence of substitutable goods and under complete information, firms offer lower prices to more central consumers, and firms' profits
} 
often try to influence the network formation, for instance by trying to create opinion leaders or by fostering social relations. In this respect, it would be interesting to explore how network information affects optimal firms' strategies. Finally, information can sometimes be designed. ${ }^{24}$ Which information structure to select, and/or how to incite agents to invest in information acquisition, is an interesting issue, which goes far beyond the objective of the present paper and is left for future research.

\section{Appendix}

\subsection{Appendix A: The game of incomplete information}

Proof of Proposition 1. We consider $W \in\{G, H\}$. For clarity, we write $P, X$ for $P(W), X(W)$. Assumptions 1 and 2 guarantee the existence of a unique and interior consumption profile. We provide now a characterization of the equilibrium (these results are known - see Candogan et al [2012]).

The first-order conditions associated with consumers' utilities, for price $P$, give the relationship between optimal consumer consumption levels and prices:

$$
P=A-(I-\delta W) X
$$

The monopoly's profit is written

$$
\Pi(W)=(P-C)^{T} X
$$

Plugging equation (3) into equation (4), we get

$$
\Pi(W)=\left[(A-C)^{T}-X^{T}(I-\delta W)^{T}\right] X
$$

The first order conditions with respect to $X$ give

$$
X=\frac{1}{2}\left(I-\delta \frac{W+W^{T}}{2}\right)^{-1}(A-C)
$$

Plugging $X$ into the profit and exploiting $X^{T}(I-\delta W)^{-T} X=X^{T}((I-$ $\left.\delta \frac{W+W^{T}}{2}\right)^{-1} X$, we find

$$
\Pi(W)=\frac{1}{4}(A-C)^{T}\left(I-\delta \frac{W+W^{T}}{2}\right)^{-1}(A-C)
$$

can decrease when network effects are higher.

${ }^{24}$ See Morris and Shin (2002), Bergemann and Pesendorfer (2007), Gentzkow and Kamenica (2011), Bergemman and Morris (2013). 
i.e.,

$$
\Pi(W)=\frac{1}{2}(A-C)^{T} X
$$

We turn to consumer surplus. First, we note that, when agent $i$ plays her best-response consumption $x_{i}^{B R}$ to others' choices and to the given price, her utility is written $u_{i}^{B R}=\frac{1}{2} \cdot\left(x_{i}^{B R}\right)^{2}$. Summing all utilities, we find

$$
C S(W)=\frac{1}{2} X^{T} X
$$

Plugging equation (6) into equation (8), we find

$$
C S(W)=\frac{1}{8}(A-C)^{T}\left(I-\delta \frac{W+W^{T}}{2}\right)^{-2}(A-C)
$$

and we are done.

Outcomes in the game of incomplete information. By construction, we have $Y=\frac{1}{2}\left(I-\delta \frac{H+H^{T}}{2}\right)^{-1}(A-C)$.

- Outcomes on symmetric networks. We start by determining the matrix $M_{H}=(I-\delta H)^{-1}$. Basically, we have

$$
M_{H}=I+\sum_{k=1}^{\infty}\left(\frac{\delta}{g}\right)^{k}\left(D D^{T}\right)^{k}
$$

Since $\left(D D^{T}\right)^{k}=\left(D^{T} D\right)^{k-1} D D^{T}$, we get

$$
M_{H}=I+\frac{\delta}{1-\frac{\delta}{g} D^{T} D} H
$$

Then we get quantities, profit and consumer surplus.

- Quantities: we have $Y=\frac{1}{2} M_{H}(A-C)$, so, using equation (9) we obtain

$$
Y=\frac{1}{2}(A-C)+\frac{D^{T}(A-C)}{2 g} \frac{\delta}{1-\frac{\delta}{g} D^{T} D} D
$$

That is,

$$
Y=\frac{A-C}{2}+\nu D
$$

with

$$
\nu=\frac{\delta}{2} \frac{D^{T}(A-C)}{g-\delta D^{T} D}
$$


- Profit: we have $\Pi(H)=\frac{1}{4}(A-C)^{T} M_{H}(A-C)$. Using equations (9) and (11), we find

$$
\Pi(H)=\frac{1}{4}(A-C)^{T}(A-C)+\frac{\delta}{2} \nu D^{T}(A-C)
$$

- Consumer surplus: we have $C S(H)=\frac{1}{8}(A-C)^{T} M_{H}^{2}(A-C)$. By equation (9) we have

$$
M_{H}^{2}=I+\phi D D^{T}
$$

where

$$
\phi=\frac{4 \nu}{\left(D^{T}(A-C)\right)^{2}} D^{T}(A-C+\nu D)
$$

We conclude that

$$
C S(H)=\frac{1}{8}(A-C)^{T}(A-C)+2 \nu D^{T}(A-C+\nu D)
$$

- Outcomes on asymmetric networks. The above results generalize to asymmetric networks as follows. Consider a network $G$ with in-degree profile $K=G \mathbf{1}$ and out-degree profile $L=G^{T} \mathbf{1}$. We define for convenience $\tau_{k}=$ $\sum_{p} k_{p}^{2}, \tau_{l}=\sum_{p} l_{p}^{2}, \tau_{k l}=\sum_{p} k_{p} l_{p}$.

We start by showing that the vector of consumptions on network $H$ is written:

$$
Y=\frac{A-C}{2}+\nu_{K} K+\nu_{L} L
$$

with

$$
\nu_{K}=\frac{\delta}{2 g} \frac{\left(1-\frac{\delta}{2 g} \tau_{k l}\right)\left(\frac{A-C}{2}\right)^{T} L+\frac{\delta}{2 g} \tau_{l}\left(\frac{A-C}{2}\right)^{T} K}{\left(1-\frac{\delta}{2 g} \tau_{k l}\right)^{2}-\left(\frac{\delta}{2 g}\right)^{2} \tau_{l} \tau_{k}}
$$

and

$$
\nu_{L}=\frac{\delta}{2 g} \frac{\left(1-\frac{\delta}{2 g} \tau_{k l}\right)\left(\frac{A-C}{2}\right)^{T} K+\frac{\delta}{2 g} \tau_{k}\left(\frac{A-C}{2}\right)^{T} L}{\left(1-\frac{\delta}{2 g} \tau_{k l}\right)^{2}-\left(\frac{\delta}{2 g}\right)^{2} \tau_{l} \tau_{k}}
$$

Proof. Denoting $\beta=\sum_{p \in N} l_{p} y_{p}$ and $\gamma=\sum_{p \in N} k_{p} y_{p}$, consumer $i$ 's consumption level satisfies

$$
y_{i}-\frac{\delta}{2 g}\left(\beta k_{i}+\gamma l_{i}\right)=\frac{a_{i}-c}{2}
$$

Exploiting this set of first-order conditions, we get

$$
\beta\left(1-\frac{\delta}{2 g} \tau_{k l}\right)-\gamma \frac{\delta}{2 g} \tau_{l}=\left(\frac{A-C}{2}\right)^{T} L
$$


and

$$
\gamma\left(1-\frac{\delta}{2 g} \tau_{k l}\right)-\beta \frac{\delta}{2 g} \tau_{k}=\left(\frac{A-C}{2}\right)^{T} K
$$

So, in the end,

$$
\beta=\frac{\left(1-\frac{\delta}{2 g} \tau_{k l}\right)\left(\frac{A-C}{2}\right)^{T} L+\frac{\delta}{2 g} \tau_{l}\left(\frac{A-C}{2}\right)^{T} K}{\left(1-\frac{\delta}{2 g} \tau_{k l}\right)^{2}-\left(\frac{\delta}{2 g}\right)^{2} \tau_{l} \tau_{k}}
$$

and

$$
\gamma=\frac{\left(1-\frac{\delta}{2 g} \tau_{k l}\right)\left(\frac{A-C}{2}\right)^{T} K+\frac{\delta}{2 g} \tau_{k}\left(\frac{A-C}{2}\right)^{T} L}{\left(1-\frac{\delta}{2 g} \tau_{k l}\right)^{2}-\left(\frac{\delta}{2 g}\right)^{2} \tau_{l} \tau_{k}}
$$

from which we get directly $y_{i}=1+\frac{\delta}{2 g}\left(\beta k_{i}+\gamma l_{i}\right)$.

We turn to profit and consumer surplus. Direct computations entail

$$
\Pi(H)=\frac{1}{4}(A-C)^{T}(A-C)+\frac{1}{2}\left(\nu_{K} K+\nu_{L} L\right)^{T}(A-C)
$$

and

$$
C S(H)=\frac{1}{8}(A-C)^{T}(A-C)+\frac{1}{2}\left(\nu_{K} K+\nu_{L} L\right)^{T}\left(\left(\nu_{K} K+\nu_{L} L+A-C\right)\right.
$$

\subsection{Appendix B: Proofs}

Proof of Proposition 2. For a given symmetric network $G$, coefficient $r_{Z, Z^{\prime}}(G)$ is written

$$
r_{Z, Z^{\prime}}(G)=\frac{1}{\sigma_{a} \sigma_{b}} \sum_{z \in \mathcal{C}_{1}, z^{\prime} \in \mathcal{C}_{2}} z z^{\prime}\left(e_{z z^{\prime}}-a_{z} b_{z^{\prime}}\right)
$$

that is,

$$
r_{Z, Z^{\prime}}(G)=\frac{1}{\sigma_{a} \sigma_{b}}\left(\sum_{i, j \in N} \frac{g_{i j} z_{i} z_{j}^{\prime}}{g}-\left(\sum_{i, j \in N} \frac{g_{i j} z_{i}}{g}\right)\left(\sum_{i, j \in N} \frac{g_{i j} z_{j}^{\prime}}{g}\right)\right)
$$

or equivalently

$$
r_{Z, Z^{\prime}}(G)=\frac{1}{\sigma_{a} \sigma_{b}}\left(\frac{Z^{T} G Z^{\prime}}{g}-\left(\frac{Z^{T} K}{g}\right)\left(\frac{L^{T} Z^{\prime}}{g}\right)\right)
$$


Letting matrix $H=\frac{K L^{T}}{g}$, we obtain finally

$$
r_{Z, Z^{\prime}}(G)=\frac{1}{\sigma_{a} \sigma_{b}} \frac{Z^{T} \Theta Z^{\prime}}{g}
$$

and we are done.

We present two useful lemmatas. Assume a symmetric network $G$ and let us define $M=(I-\delta G)^{-1}$. The respective consumption profiles under complete and incomplete information are $X=\frac{1}{2}(I-\delta G)^{-1}(A-C)$ and $Y=\frac{1}{2}(I-\delta H)^{-1}(A-C)$. For all vector $V$, We let $\|V\|_{M}=\sqrt{V^{T} M V}$ denote the $M$-norm of vector $V$ (this is a norm by symmetry of matrix $M$ ), and we let $\|V\|$ represent the euclidian norm.

Lemma 1. We have

$$
\Pi(G)-\Pi(H)=\delta^{2}\|\Theta Y\|_{M}^{2}+\delta Y^{T} \Theta Y
$$

Lemma 2. We have

$$
C S(G)-C S(H)=\frac{\delta^{2}}{2}\|M \Theta Y\|^{2}+\delta Y^{T} M \Theta Y
$$

Proof of Lemma 1. We have

$$
(I-\delta G) X=(I-\delta H) Y
$$

that is,

$$
(I-\delta G)(X-Y)=\delta \Theta Y
$$

or equivalently, given that $M=(I-\delta G)^{-1}$,

$$
X=Y+\delta M \Theta Y
$$

Thus,

$$
\frac{1}{2}(A-C)^{T}(X-Y)=\delta X^{T} \Theta Y
$$

that is

$$
\Pi(G)-\Pi(H)=\delta X^{T} \Theta Y
$$

Plugging equation (19) into equation (20), we obtain

$$
\Pi(G)-\Pi(H)=\delta Y^{T} \Theta Y+\delta^{2} Y^{T} \Theta^{T} M \Theta Y
$$


and we are done.

Proof of Lemma 2. Proof. Recalling that $X=Y+\delta M \Theta Y$, we deduce that

$$
X^{T} X-Y^{T} Y=\delta^{2}(M \Theta Y)^{T}(M \Theta Y)+2 \delta Y^{T} M \Theta Y
$$

The gap in consumer surplus being $C S(G)-C S(H)=\frac{1}{2}\left(X^{T} X-Y^{T} Y\right)$, we obtain

$$
C S(G)-C S(H)=\frac{\delta^{2}}{2}\|M \Theta Y\|^{2}+\delta Y^{T} M \Theta Y
$$

and we are done.

Proof of Theorem 1. We consider a non-regular network $G$. We recall that $A=\mathbf{1}, X=\frac{1-c}{2} B(G)$, and $Y=\frac{1-c}{2}\left[\mathbf{1}+\left(\frac{\delta}{1-\delta \frac{D^{T} D}{g}}\right) D\right]$.

- If $\Theta D \neq \mathbf{0}$ :

I. Monopoly's profit.

We prove that the condition $r_{D}(G) \geq 0$ is sufficient:

Note that $\Theta D \neq \mathbf{0}$ implies $\Theta Y \neq \mathbf{0}$. By equation (17) in Lemma 1, $\Pi(G)-\Pi(H)>0$ if

$$
Y^{T} \Theta Y \geq 0
$$

Plugging equation (10) into condition (21) and recalling that $\Theta \mathbf{1}=\mathbf{0}$, we get $D^{T} \Theta D \geq 0$, which is equivalent to $r_{D}(G) \geq 0$.

We prove that the condition $r_{D}(G) \geq 0$ is also necessary:

Suppose that $r_{D}(G)<0$. We introduce $e=\frac{1-c}{2}$ for convenience. We have, for $\delta$ small enough,

$$
\begin{aligned}
& \frac{1}{e} X=\mathbf{1}+\delta G \mathbf{1}+\delta^{2} G^{2} \mathbf{1}++\delta^{3} G^{3} \mathbf{1}+o\left(\delta^{3}\right) \\
& \frac{1}{e} Y=\mathbf{1}+\delta H \mathbf{1}+\delta^{2} H^{2} \mathbf{1}++\delta^{3} H^{3} \mathbf{1}+o\left(\delta^{3}\right)
\end{aligned}
$$

We recall that $\Pi(G)-\Pi(H)=e(X-Y)$, and that, because networks $G$ and $H$ have same degree profile, $\Theta \mathbf{1}=\mathbf{0}$ and $\mathbf{1}^{T}\left(G^{2}-H^{2}\right) \mathbf{1}=\mathbf{0}$. We thus obtain

$$
\Pi(G)-\Pi(H)=e^{2} \delta^{3} \mathbf{1}^{T}\left(G^{3}-H^{3}\right) \mathbf{1}+o\left(\delta^{3}\right)
$$


Now, we observe that, because $G \mathbf{1}=H \mathbf{1}$, we have

$$
\mathbf{1}^{T}\left(G^{3}-H^{3}\right) \mathbf{1}=D^{T} \Theta D
$$

By Proposition 2, $r_{D}(G)<0$ involves $D^{T} \Theta D<0$, and therefore $\Pi(G)-$ $\Pi(H)<0$.

II. Consumer surplus.

We prove that the condition $r_{D}(G) \geq 0$ is sufficient:

By equation (10), $Y=\frac{1-c}{2} \mathbf{1}+\nu G \mathbf{1}$. Hence,

$$
Y^{T} M \Theta Y=\frac{1-c}{2} \mathbf{1}^{T} M \Theta Y+\nu \mathbf{1}^{T} G M \Theta Y
$$

We note that

$$
\frac{1-c}{2} \mathbf{1}^{T} M \Theta Y=X^{T} \Theta Y
$$

Moreover, because $X-\frac{1-c}{2} \mathbf{1}=\delta G M \mathbf{1}$ and $G M=M G$ (since $G^{T}=G$ ), we have

$$
\mathbf{1}^{T} G M \Theta Y=\frac{1}{\delta}\left(X-\frac{1-c}{2} \mathbf{1}\right)^{T} \Theta Y
$$

In the end,

$$
Y^{T} M \Theta Y=X^{T} \Theta Y+\frac{\nu}{\delta}\left(X-\frac{1-c}{2} \mathbf{1}\right)^{T} \Theta Y
$$

Exploiting now that $\Theta^{T} \mathbf{1}=\Theta \mathbf{1}=\mathbf{0}$ (where $\mathbf{0}$ represents the vector with entries equal to 0$)$, we obtain

$$
\delta Y^{T} M \Theta Y=(\nu+\delta) X^{T} \Theta Y
$$

Now, by equation (20) we have

$$
X^{T} \Theta Y=\frac{\Pi(G)-\Pi(H)}{\delta}
$$

Thus, combining equations (18), (22) and (23), we get

$$
C S(G)-C S(H)=\frac{\delta^{2}}{2}\|M \Theta Y\|^{2}+\left(1+\frac{\nu}{\delta}\right)(\Pi(G)-\Pi(H))
$$

where $\nu>0$. Since $\Theta Y \neq \mathbf{0}$, we have $M \Theta Y \neq \mathbf{0}$. The condition $r_{D}(G) \geq 0$ involves a positive profit gap. This entails a positive consumer surplus gap. 
We prove that the condition $r_{D}(G) \geq 0$ is also necessary:

Suppose first that $r_{D}(G)<0$. Reacalling that $e=\frac{1-c}{2}$ and that $C S(G)-$ $C S(H)=\frac{1}{2}\left(X^{T} X-Y^{T} Y\right)$, we obtain

$\frac{1}{e^{2}}(C S(G)-C S(H))=\delta \mathbf{1}^{T} \Theta \mathbf{1}+\frac{3}{2} \delta^{2} \mathbf{1}^{T}\left(G^{2}-H^{2}\right) \mathbf{1}+\frac{3}{2} \delta^{3} \mathbf{1}^{T}\left(G^{3}-H^{3}\right) \mathbf{1}+o\left(\delta^{3}\right)$

And because $\Theta \mathbf{1}=\mathbf{0}$, we get

$$
C S(G)-C S(H)=\frac{3 e^{2}}{2} \delta^{3} \mathbf{1}^{T}\left(G^{3}-H^{3}\right) \mathbf{1}+o\left(\delta^{3}\right)
$$

Note that, because $G \mathbf{1}=H \mathbf{1}$, we have

$$
\mathbf{1}^{T}\left(G^{3}-H^{3}\right) \mathbf{1}=D^{T} \Theta D
$$

and thus, $r_{D}(G)<0$ implies $C S(G)-C S(H)<0$.

- If $\Theta D=\mathbf{0}$, we have that $\Theta Y=\Theta(\mathbf{1}+\nu D)=\mathbf{0}$. By equation (19), we have that $X=Y$. Thus we obtain that $\Pi(G)=\Pi(H)$ and $C S(G)=C S(H)$.

Proof of Proposition 3. We observe from equation (19) that

$$
\left.\mathbf{1}^{T}(X-Y)>0 \text { iff }-\delta \| \Theta Y\right) \|_{M}^{2}<Y^{T} \Theta Y
$$

(with $\| Z) \|_{M}^{2}=Z^{T} M Z$ ) We note that $M$ is a symmetric positive definite matrix, with a spectrum deduced from the spectrum of $G$ as follows: if $\lambda_{i}$ is an eigenvalue of $G$, then $\frac{1}{1-\delta \lambda_{i}}$ is a positive eigenvalue of $M$. Letting $\lambda_{\min }(G)$ denote the smallest eigenvalue of $G$, the following standard inequality applies:

$$
\forall Z \neq \mathbf{0}, Z^{T} M Z \geq \frac{1}{1-\delta \lambda_{\min }(G)} Z^{T} Z
$$

It follows that $\mathbf{1}^{T}(X-Y)>0$ if

$$
-\left(\frac{\delta}{1-\lambda_{\min }(G)}\right)\|\Theta Y\|^{2}<Y^{T} \Theta Y
$$

Since $Y=\frac{1-c}{2} \mathbf{1}+\nu D$ and $\Theta \mathbf{1}=\mathbf{0}$, we have

$$
\Theta Y=\nu \Theta D
$$


Because $\Theta D \neq \mathbf{0}$, the problem is non-degenerated (otherwise the inequality (25) is impossible, we would have $\Theta Y=\mathbf{0}$ ). Noting that

$$
\sum_{i}\left(\sum_{j} g_{i j} d_{j}\right)^{2}=\left\|G^{2} \mathbf{1}\right\|^{2}=\mathbf{1}^{T} G^{4} \mathbf{1}=g^{(4)}
$$

we obtain

$$
\|\Theta Y\|^{2}=\nu^{2}\left(\frac{\left(g^{(2)}\right)^{3}}{g^{2}}-2 \frac{g^{(2)} g^{(3)}}{g}+g^{(4)}\right)
$$

Exploiting $Y^{T} \Theta Y=r_{D}(G)\left(\sum_{i} d_{i}^{3}-\frac{\left(g^{(2)}\right)^{2}}{g}\right)$, we obtain

$$
r_{D}(G)>-\left(\frac{\delta \nu^{2}}{1-\delta \lambda_{\min }(G)}\right)\left(\frac{g^{2} g^{(4)}+\left(g^{(2)}\right)^{3}-2 g g^{(2)} g^{(3)}}{g \sum_{i} d_{i}^{3}-\left(g^{(2)}\right)^{2}}\right)
$$

To finish, from equation (24), increased profit entails increased consumer surplus.

Proof of Corollary 1. We differentiate the system of first order conditions $(I-\delta G) X=\frac{1-c}{2} \mathbf{1}$ with respect to $\delta$. We denote by $X^{\prime}$ the derivative of vector $X$ with respect to parameter $\delta$. We obtain:

$$
(I-\delta G) X^{\prime}=G X
$$

that is, with $M=(I-\delta G)^{-1}$,

$$
X^{\prime}=M G X
$$

Thus, we get

$$
\mathbf{1}^{T} X^{\prime}=\frac{2}{1-c} X^{T} G X
$$

Given that $\delta G X=X-\frac{1-c}{2} \mathbf{1}$, we obtain

$$
\delta \mathbf{1}^{T} X^{\prime}=\frac{2}{1-c} X^{T} X-\mathbf{1}^{T} X
$$

and since $C S(G)=\frac{X^{T} X}{2}$ and $\Pi(G)=\frac{1-c}{2} \mathbf{1}^{T} X$,

$$
\delta \mathbf{1}^{T} X^{\prime}=\frac{2}{1-c}(2 C S(G)-\Pi(G))
$$

Hence, we are in position to characterize the sign of the variation in profit gap with $\delta$. Indeed:

$$
\frac{(1-c) \delta}{2} \mathbf{1}^{T}\left(X^{\prime}-Y^{\prime}\right)=2(C S(G)-C S(H))-(\Pi(G)-\Pi(H))
$$


By equation (24), and given that $\Theta D \neq \mathbf{0}, C S(G)-C S(H)>\Pi(G)-\Pi(H)$, so that

$$
\frac{(1-c) \delta}{2} \mathbf{1}^{T}\left(X^{\prime}-Y^{\prime}\right)>\Pi(G)-\Pi(H)
$$

and since $r_{D}(G) \geq 0$, we have $\Pi(G)-\Pi(H)>0$, so $\mathbf{1}^{T}\left(X^{\prime}-Y^{\prime}\right)>0$.

Proof of Theorem 2. We let $e=\frac{1-c}{2}$. We let $X=e(I-\delta \tilde{G})^{-1} \mathbf{1}$ and $Y=e\left(I-\delta \frac{H+H^{T}}{2}\right)^{-1} \mathbf{1}$ represent respectively the equilibrium demand profiles in the games of respectively complete and incomplete information.

We start with the monopoly's profit. By Proposition $1, \Pi(G)=\Pi(\tilde{G})$. We let matrix $\tilde{H}=\frac{\tilde{D} \tilde{D}^{T}}{g}$ denote the matrix of interaction of the game with incomplete information on network $\tilde{G}$. By Theorem $1,(\tilde{G}-\tilde{H}) \tilde{D} \neq \mathbf{0}$ and $r_{\tilde{D}}(\tilde{G}) \geq 0$ involve $\Pi(\tilde{G})>\Pi(\tilde{H})$. It is therefore sufficient to show that $\Pi(\tilde{H}) \geq \Pi\left(\frac{H+H^{T}}{2}\right)$ for all $\delta$. Now, define $M_{\tilde{H}}=(I-\delta \tilde{H})^{-1}$. Similar simple computations as in Lemma 1 entail

$$
\Pi(\tilde{H})-\Pi\left(\frac{H+H^{T}}{2}\right)=\delta^{2}\left\|\left(\tilde{H}-\frac{H+H^{T}}{2}\right) Y\right\|_{M_{\tilde{H}}}^{2}+\delta Y^{T}\left(\tilde{H}-\frac{H+H^{T}}{2}\right) Y
$$

Recalling that $\frac{H+H^{T}}{2}=\frac{K L^{T}+L K^{T}}{2 g}$, few computations entail

$$
\tilde{H}-\frac{H+H^{T}}{2}=\frac{1}{4 g}(L-K)(L-K)^{T}
$$

This means that matrix $\tilde{H}-\frac{H+H^{T}}{2}$ is positive definite. Therefore, we have $Y^{T}\left(\tilde{H}-\frac{H+H^{T}}{2}\right) Y>0$, which shows the result.

We turn to consumer surplus. We use the same overall strategy as with monopoly profit. First we note that $C S(G)=C S(\tilde{G})$. Moreover, $(\tilde{G}-\tilde{H}) \tilde{D} \neq \mathbf{0}$ and $r_{\frac{K+L}{2}}(\tilde{G}) \geq 0$ implies $C S(\tilde{G})>C S(\tilde{H})$. It is therefore sufficient to show that $C S(\tilde{H}) \geq C S\left(\frac{H+H^{T}}{2}\right)$.

To proceed, we examine the sum of squares of centralities given by equation (14). We write $\alpha=\nu_{\tilde{D}}, \beta=\nu_{K}, \gamma=\nu_{L}$ for convenience. The square of consumer $i$ 's consumption for network $\tilde{H}$ is

$$
\left[1+\delta \alpha\left(k_{i}+l_{i}\right)\right]^{2}=1+2 \delta \alpha\left(k_{i}+l_{i}\right)+\delta^{2} \alpha^{2}\left(k_{i}^{2}+l_{i}^{2}+2 k_{i} l_{i}\right)
$$

while the square of agent $i$ 's consumption for network $H$ is

$$
\left[1+\delta \beta k_{i}+\delta \gamma l_{i}\right]^{2}=1+2 \delta \beta k_{i}+2 \delta \gamma l_{i}+\delta^{2}\left(\beta^{2} k_{i}^{2}+\gamma^{2} l_{i}^{2}+2 \beta \gamma k_{i} l_{i}\right)
$$


Summing over all agents, it is sufficient to show that all terms before the quantities $\tau_{k l}, \tau_{l}, \tau_{k}$ are larger in the former situation than in the latter. We get:

- for $\tau_{k l}$, it must be that $\gamma \beta \leq \alpha^{2}$. But we know that $\frac{\gamma+\beta}{2} \leq \alpha$, and it is true that $\gamma \beta \leq\left(\frac{\gamma+\beta}{2}\right)^{2}$ because this means $(\beta-\gamma)^{2} \geq 0$.

- for $\tau_{k}$ and $\tau_{l}$, it is sufficient to prove that $\tau_{k}\left(\alpha^{2}-\beta^{2}\right)+\tau_{l}\left(\alpha^{2}-\gamma^{2}\right) \geq 0$. Now, exploiting that $\frac{\gamma+\beta}{2} \leq \alpha$, we have

$$
\tau_{k}\left(\alpha^{2}-\beta^{2}\right)+\tau_{l}\left(\alpha^{2}-\gamma^{2}\right) \geq \frac{\gamma-\beta}{4}\left[(\gamma+3 \beta) \tau_{k}-(\beta+3 \gamma) \tau_{l}\right]
$$

Assume w.l.o.g. that $\gamma \geq \beta$, that is $\tau_{k} \geq \tau_{l}$. Then we have

$$
\tau_{k}\left(\alpha^{2}-\beta^{2}\right)+\tau_{l}\left(\alpha^{2}-\gamma^{2}\right) \geq \frac{\gamma-\beta}{4}\left[(\gamma+3 \beta) \tau_{k}-(\beta+3 \gamma) \tau_{l}\right]
$$

Now, we have $\beta \tau_{k}-\gamma \tau_{l} \geq 0$ : replacing $\beta$ and $\gamma$, we get that this quantity is proportional to $\tau_{k}-\tau_{l}$. Hence,

$$
\tau_{k}\left(\alpha^{2}-\beta^{2}\right)+\tau_{l}\left(\alpha^{2}-\gamma^{2}\right) \geq 0
$$

We thus obtain $C S(\tilde{H}) \geq C S\left(\frac{H+H^{T}}{2}\right)$.

Proof of Proposition 4. We introduce $E=\frac{A-C}{2}$ for convenience. We have, for $\delta$ small enough,

$$
\begin{gathered}
X=E+\delta G E+\delta^{2} G^{2} E+\delta^{3} G^{3} E+o\left(\delta^{3}\right) \\
Y=E+\delta H E+\delta^{2} H^{2} E+\delta^{3} H^{3} E+o\left(\delta^{3}\right)
\end{gathered}
$$

Recall that $\Pi(G)-\Pi(H)=E^{T}(X-Y)$ and $C S(G)-C S(H)=\frac{1}{2}\left(X^{T} X-\right.$ $\left.Y^{T} Y\right)$. Noting that $E^{T} G H E=E^{T} H G E\left(\right.$ so $E^{T}\left(G^{2}-H^{2}\right) E=E^{T}(G+$ $H) \Theta E$, we obtain

$$
\begin{array}{r}
\Pi(G)-\Pi(H)=\delta E^{T} \Theta E+\delta^{2} E^{T}(G+H) \Theta E+\delta^{3} E^{T}\left(G^{3}-H^{3}\right) E+o\left(\delta^{3}\right) \\
C S(G)-C S(H)=2 \delta E^{T} \Theta E+3 \delta^{2} E^{T}(G+H) \Theta E+4 \delta^{3} E^{T}\left(G^{3}-H^{3}\right) E+o\left(\delta^{3}\right)
\end{array}
$$

We note that $E^{T} \Theta E=A^{T} \Theta A$, which entails

$$
\begin{array}{r}
\Pi(G)-\Pi(H)=\delta A^{T} \Theta A+o(\delta) \\
C S(G)-C S(H)=2 \delta A^{T} \Theta A+o(\delta)
\end{array}
$$

Thus, when $r_{A}(G)>0$, i.e. $A^{T} \Theta A>0$, outcome gaps are positive. 


\section{Proof of Theorem 3.}

- $\Theta A \neq \mathbf{0}$ or $\Theta D \neq \mathbf{0}$ : in this case $\Theta Y=\Theta(A+\nu D) \neq \mathbf{0}$, so $\|\Theta Y\|_{M}>0$. Then, by Lemma 1, we have that

$$
Y^{T} \Theta Y \geq 0 \text { implies } \Pi(G)-\Pi(H)>0
$$

We exploit $Y=\frac{A-C}{2}+\nu D$ (see equation (10)). Define

$$
f(t)=\left(\frac{A-C}{2}+t D\right)^{T} \Theta\left(\frac{A-C}{2}+t D\right)
$$

The sufficient condition is thus expressed as $f(\nu) \geq 0$. Note that parameter $\nu$ is increasing with $\delta$, and when $\delta$ goes from 0 to its maximal bound, $\nu$ goes from 0 to infinity.

Now, $r_{A}(G) \geq 0$ implies $f(0) \geq 0$. Moreover, $r_{D}(G) \geq 0$ implies $f(+\infty) \geq$ 0 . For intermediate values of parameter $\nu$, note that $r_{D}(G) \geq 0$ entails that $f($.$) is U-shaped. The minimum is attained at \nu^{*}=\frac{-\left(\frac{A-C}{2}\right)^{T} \Theta D}{D^{T} \Theta D}$, and $f\left(\nu^{*}\right) \geq 0$ if $r_{A, D}(G) \geq-\sqrt{r_{A}(G) \cdot r_{D}(G)}$.

- When $\Theta A=\Theta D=\mathbf{0}$, we have $\Theta Y=\mathbf{0}$. Lemma 1 then involves $\Pi(G)=\Pi(H)$.

\section{Proof of Theorem 4.}

- $\Theta A \neq \mathbf{0}$ or $\Theta D \neq \mathbf{0}$ : assume that $r_{D}(G) \geq 0, r_{A}(G) \geq 0, r_{A, D}(G) \geq$ $-\sqrt{r_{A}(G) \cdot r_{D}(G)}$, and $r_{B(G), A}(G) \geq 0$.

We have $\Theta Y=\Theta(A+\nu D) \neq \mathbf{0}$, so $\|M \Theta Y\|>0$. Then, by Lemma 2, $Y^{T} M \Theta Y>0$ guarantees that $C S(G)>C S(H)$. We will show that $Y^{T} M \Theta Y>0$. Since $Y^{T} M \Theta Y=X^{T} \Theta Y+\nu \mathbf{1}^{T} G M \Theta Y$, we will show that $X^{T} \Theta Y>0$ and $\mathbf{1}^{T} G M \Theta Y>0$.

- $X^{T} \Theta Y>0$ : recall that $\Pi(G)-\Pi(H)=\delta X^{T} \Theta Y$. Therefore, under the three first conditions, $X^{T} \Theta Y>0$.

. $\mathbf{1}^{T} G M \Theta Y>0$ : Note that $G M=\frac{M-I}{\delta}$, so $\mathbf{1}^{T} G M \Theta Y>0$ whenever $B(G)^{T} \Theta Y>0($ with $B(G)=M 1)$.

Since $Y=(A-C) / 2+\nu D, B(G)^{T} \Theta Y>0$ is implied by $B(G)^{T} \Theta A>0$ and $B(G)^{T} \Theta D>0$. Now, on the one hand, $r_{B(G), A}(G) \geq 0$ means $A^{T} \Theta B(G) \geq$ 0 . On the other hand, $r_{D}(G)>0$ implies $D^{T} \Theta B(G)>0$. This stems from Theorem 1: indeed, this theorem states that profit gap is positive when $D^{T} \Theta D>0$, and in the homogenous case, profit gap is proportional to $B(G)^{T} \Theta(\mathbf{1}+\nu D)$, i.e., to $B(G)^{T} \Theta D$.

Note that

$$
C S(G)<C S(H) \text { implies } B(G)^{T} \Theta A<0
$$


To see this, it follows directly from the above proof that $C S(G)<C S(H)$ implies $\mathbf{1}^{T} M \Theta Y<0$ (which is more demanding that the necessary condition $\left.Y^{T} M \Theta Y<0\right)$. Noting that $Y=(A-C) / 2+\nu D$ and remembering that $\mathbf{1}^{T} M \Theta D \geq 0$ because $r_{D}(G) \geq 0$, it follows that the condition $\mathbf{1}^{T} M \Theta Y<0$ requires $\mathbf{1}^{T} M \Theta(A-C)<0$, that is $B(G)^{T} \Theta A<0$.

- When $\Theta A=\Theta D=\mathbf{0}$, we have $\Theta Y=\mathbf{0}$. Lemma 2 then involves $C S(G)=C S(H)$.

Proof of Proposition 6. We set $c=0$ for convenience as it plays no role in the proof. We let $\bar{H}=\frac{K 1^{T}}{n}$. For convenience, we denote by $Y=B\left(\frac{\bar{H}+\bar{H}^{T}}{2}, \delta\right)$ the consumption profile under incomplete information and we define $\Phi=\frac{G+G^{T}}{2}-\frac{\bar{H}+\bar{H}^{T}}{2}$. We will first characterize $Y$. Second, we apply Lemma 1, which still holds in this scenario.

Step 1: We characterize the vector $Y$. For consumer $i$, the probability of being influenced by any consumer $j$ is $\frac{k_{i}}{n}$. Therefore, vector $Y$ satisfies

$$
y_{i}=1+\delta \sum_{j} \frac{k_{i}+k_{j}}{2 n} y_{j}
$$

Setting $y=\mathbf{1}^{T} Y$, we get after simple development

$$
y=\frac{4 n}{(2-\delta d)^{2}-\delta^{2} \sum_{i} k_{i}^{2}}
$$

and consumer $i$ 's consumption is written

$$
y_{i}=\frac{4+2 \delta\left(k_{i}-d\right)}{(2-\delta d)^{2}-\frac{\delta^{2}}{n} \sum_{p} k_{p}^{2}}
$$

Step 2: We can show as in Lemma 1 that

$$
\Pi\left(\frac{G+G^{T}}{2}\right)-\Pi\left(\frac{\bar{H}+\bar{H}^{T}}{2}\right)=\delta^{2}\|\Phi Y\|_{M}^{2}+\delta Y^{T} \Phi Y
$$

Hence, $Y^{T} \Phi Y \geq 0$ guarantees a positive profit gap. Exploiting equation (26), we find

$$
\sum_{i} \sum_{j} \phi_{i j}\left(2-\delta d+\delta k_{i}\right)\left(2-\delta d+\delta k_{j}\right) \geq 0
$$

That is, given that $\mathbf{1}^{T} \Phi \mathbf{1}=0$ and simplifying by $\delta$,

$$
2(2-\delta d) \sum_{i} \sum_{j}\left(\frac{g_{i j}+g_{j i}}{2}-\frac{k_{i}+k_{j}}{2 n}\right) k_{i}+\delta \sum_{i} \sum_{j}\left(\frac{g_{i j}+g_{j i}}{2}-\frac{k_{i}+k_{j}}{2 n}\right) k_{i} k_{j} \geq 0
$$


that is,

$$
\sum_{i} \sum_{j} \frac{g_{i j}+g_{j i}}{2} k_{i} k_{j} \geq n\left(\frac{2-\delta d}{\delta}\right)\left(d^{2}-d \sum_{i} k_{i} l_{i}\right)+\frac{1}{n} \sum_{i} k_{i}^{2}
$$

Noting that $K^{T} G K=K^{T}\left(\frac{G+G^{T}}{2}\right) K$, we get

$$
\sum_{i} \sum_{j} g_{i j} k_{i} k_{j} \geq n\left(\frac{2-\delta d}{\delta}\right)\left(d^{2}-\frac{1}{n} \sum_{i} k_{i} l_{i}\right)+d \sum_{i} k_{i}^{2}
$$

Remembering that $\sum_{i} k_{i} l_{i}=n\left(\operatorname{Cov}(k, l)+d^{2}\right)$ (where $\operatorname{Cov}(k, l)$ is the covariance between in-degrees and out-degrees), we obtain

$$
\sum_{i} \sum_{j} g_{i j} k_{i} k_{j}-d \sum_{i} k_{i}^{2}+n\left(\frac{2-\delta d}{\delta}\right) \operatorname{Cov}(k, l) \geq 0
$$

We add and substract the term $\frac{1}{g}\left(\sum_{p} k_{p}^{2}\right)\left(\sum_{p} k_{p} l_{p}\right)$, and we note that $\frac{\sum_{i} k_{i} l_{i}}{g}=d+\frac{\operatorname{Cov}(k, l)}{d}$. The above equation is then equivalent to

$$
\left[\sum_{i} \sum_{j} g_{i j} k_{i} k_{j}-\frac{1}{g}\left(\sum_{p} k_{p}^{2}\right)\left(\sum_{p} k_{p} l_{p}\right)\right]+\operatorname{Cov}(k, l)\left(\frac{\sum_{i} k_{i}^{2}}{d}+n\left(\frac{2-\delta d}{\delta}\right)\right) \geq 0(28)
$$

The first term is equal to $K^{T}\left(G-\frac{K L^{T}}{g}\right) K$ and is positive if $r_{K}(G)>0$ (in-degree assortativity). The second term is non-negative if in-degrees and out-degrees are positively correlated $\left(\rho_{k, l} \geq 0\right)$.

\section{References}

[1] Acemoglu, D., A. Malekian and A. Ozdaglar, 2013, Network Security and Contagion, NBER Working Paper No. 19174.

[2] Ballester, C., A. Calvò-Armengol and Y. Zenou, 2006, Who's who in networks. Wanted: the key player, Econometrica, vol. 74(5), 1403-1417.

[3] Becker, G., 1973, A theory of marriage: Part I, Journal of Political Economy, vol. 81, 813846.

[4] Bénabou, R., 1996, Heterogeneity, stratification, and growth: macroeconomic implications of community structure and school finance, American Economic Review, vol. 86, 584-609. 
[5] Bloch, F. and N. Quérou, 2013, Pricing in Social Networks, Games and Economic Behavior, vol. 80, 263-281.

[6] Bramoullé, Y., S. Currarini, M. Jackson, P. Pin and B. Rogers, 2012, Homophily and Long-Run Integration in Social Networks, Journal of Economic Theory, vol. 147(5), 1754-1786.

[7] Cabrales, A., P. Gottardi and F. Vega-Redondo, 2014, Risk-Sharing and Contagion in Networks, CESifo Working Paper No. 4715.

[8] Candogan, O., K. Bimpikis and A. Ozdaglar, 2012, Optimal Pricing in the Presence of Local Network Effects, Operations Research, vol. 60(4), 883-905.

[9] Charness, G., F. Feri, M. Meléndez-Jiménez and M. Sutter, 2014, Experimental Games on Networks: Underpinnings of Behavior and Equilibrium Selection, Econometrica, vol. 82(5), 1615-1670.

[10] Chen, Y.-J., Y. Zenou and J. Zhou, 2015, Competitive Pricing Strategies in Social Networks, mimeo.

[11] Currarini, S., M. Jackson and P. Pin, 2009, An Economic Model of Friendship: Homophily, Minorities and Segregation, Econometrica, vol. 77(4), 1003-1045.

[12] de Marti, J. and Y. Zenou, 2015, Network Games with Incomplete Information, Journal of Mathematical Economics, vol. 61, 221-240.

[13] Durlauf, S. and A. Seshadri, 2003, Is Assortative Matching Efficient?, Economic Theory, vol. 21, 475-493.

[14] Economides, N., 1996, The Economics of Networks, International Journal of Industrial Organization, vol. 14(6), 673-699.

[15] Erdös, P. and A. Rényi, 1960, On the Evolution of Random Graphs, Publ. Math. Inst. Hungar. Acad. Sci 5, 17-61.

[16] Fainmesser, I. and A. Galeotti, 2015, Pricing Network Effects, forthcoming in the Review of Economic Studies.

[17] Farrell, J. and G. Saloner, 1985, Standardization, Compatibility and Innovation, RAND Journal of Economics, vol. 16, 70-83.

[18] Galeotti, A., S. Goyal, M. Jackson, F. Vega-Redondo and L. Yariv, 2010, Network Games, Review of Economic Studies, , vol. 77(1), 218-244. 
[19] Galeotti, A. and B. Rogers, 2013, Strategic Immunization and Group Structure, American Economic Journal: Microeconomics, vol. 5(2), 132 .

[20] Hagberg A and N. Lemons N, 2015, Fast Generation of Sparse Random Kernel Graphs, PLoS ONE 10(9): e0135177.

[21] Hemmecke, R., S. Kosub, E. Mayr, H. Täubig and J. Weihmann, 2011, Inequalities for the Number of Walks in Trees and General Graphs and a Generalization of a Theorem of Erdös and Simonovits, mimeo Technische Universität München.

[22] Jackson, M. and B. Rogers, 2007, Meeting Strangers and Friends of Friends: How Random Are Social Networks?, American Economic Review, vol. 97(3), 890-915.

[23] Jackson, M. and L. Yariv, 2005, Diffusion on Social Networks, Economie Publique, 16(1), 3-16.

[24] Jackson, M. and L. Yariv, 2007, Diffusion of Behavior and Equilibrium Properties in Network Games, American Economic Review, vol. 93(2), 92-98.

[25] Katz, M. and C. Shapiro, 1985, Network Externalities, Competition and Compatibility, American Economic Review, vol. 75, 424-440.

Marketing Research Symposium.

[26] Kets, W., 2011, Robustness of Equilibria in Anonymous Local Games, Journal of Economic Theory, vol. 146(1), 300-325.

[27] König, M., C. Tessone and Y. Zenou, From Assortative to Dissortative Networks: The Role of Capacity Constraints, Advances in Complex Systems, Vol. 13(4), 483-499.

[28] Lazarsfeld, P. and R. Merton, 1954, Friendship as a social process: A substantive and methodological analysis. In Freedom and control in modern society, ed. M. Berger, T. Abel, and C. Page, 18-66, New York: Van Nostrand.

[29] Li, L., D. Alderson, W. Willinger, J. Doyle., 2004, A first-principles approach to understanding the internets router-level topology, ACM SIGCOMM Computer Communication Review, vol. 34(4), 3-14. 
[30] McPherson, M., L. Smith-Lovin and J. Cook, 2001, Birds of a Feather: Homophily in Social Networks, American Review of Sociology, vol. 27, 415-444.

[31] Molloy, M. and B. Reed, 1995, A Critical Point for Random Graphs with a Given Degree Sequence, Random Structures \& Algorithms, vol. 6(2-3), 161-180.

[32] Newman, 2002, Assortative Mixing in Networks, Physical Review Letters, vol 89(20), 208701.

[33] Pin, P. and F. Feri, 2016, The Effect of Externalities Aggregation on Network Games Outcomes, mimeo.

[34] Pin, P. and B. Rogers, 2015, Stochastic Network Formation and Homophily, forthcoming in the The Oxford Handbook on the Economics of Networks.

[35] Pirhaveenan, M., M. Prokopenko and A. Zomaya, 2009, Assortative Mixing in Directed Networks, Technical Report 637, University of Sydney.

[36] Serrano, M., M Boguñá, R. Pastor-Satorras and A. Vespignani, 2007, Correlations in complex networks, Structure and Dynamics of Complex Networks. From Information Technology to Finance and Natural Science World Scientific, Singapur.

[37] Sundararajan, A., 2008, Local Network Effects and Network Structure, B.E. Journal of Theoretical Economics, vol. 7(1).

[38] Peter, R., A. Richter and P. Thistle, 2014, Endogenous Information, Advers e Selection, and Prevention: Implications for Genetic Testing Policy, mimeo. 